Chapter 14

\title{
Targeted Lentiviral Vectors: Current Applications and Future Potential
}

\author{
Cleo Goyvaerts, Therese Liechtenstein, \\ Christopher Bricogne, David Escors and \\ Karine Breckpot \\ Additional information is available at the end of the chapter \\ http://dx.doi.org/10.5772/52770
}

\section{Introduction}

About two decades ago recombinant human immunodeficiency virus type 1 (HIV-1) was proposed as a blueprint for the development of lentiviral vectors (LVs) (Naldini, Blomer et al. 1996). Lentiviral vectors exhibit several characteristics that make them favorable tools for gene therapy, including sustained gene delivery through vector integration, transduction of both dividing and non-dividing cells, applicability to different target cell types, absence of expression of viral proteins after transduction, delivery of complex genetic elements, low genotoxicity and the relative ease of vector manipulation and production (Cattoglio, Facchini et al. 2007; Bauer, Dao et al. 2008). This is reflected in the numerous applications such as: transgene (tg) overexpression (Lopez-Ornelas, Mejia-Castillo et al. 2011), persistent gene silencing (Wang, Hu et al. 2012), immunization (Breckpot, Emeagi et al. 2008), generation of transgenic animals (Baup, Fraga et al. 2010), in vivo imaging (Roet, Eggers et al. 2012), induction of pluripotent cells, stem cell modification (Sanchez-Danes, Consiglio et al. 2012), lineage tracking and site-directed gene editing (Lombardo, Genovese et al. 2007) as well as many applications targeting cancer cells (Petrigliano, Virk et al. 2009).

Recombinant LVs can be derived from primate as well as non-primate lentiviruses such as HIV-1 and simian immunodeficiency virus (SIV) next to the equine infectious anemia virus, caprine arthritis-encephalitis virus, maedi-visna virus, feline immunodeficiency virus (FIV) and bovine immunodeficiency virus respectively (Escors and Breckpot 2010). They are all members of the Retroviridae family with 'retro' referring to their capacity to retro-transcribe their diploid single stranded (ss) RNA genome into a double stranded (ds) DNA copy that is 
integrated in the genome of the infected host cell (Figure 1A). Since LVs are most often derived from HIV-1, the generation of recombinant LVs has been accompanied by several safety concerns such as the generation of replication-competent lentiviruses (RCLs). Another potential biosafety concern is the induction of insertional mutagenesis, a major genotoxic problem that emerged in gene therapy clinical trials using their $\gamma$-retroviral counterparts (Manilla, Rebello et al. 2005). Generally, LVs are produced by transiently transfecting HEK 293 or 293T cells with plasmids encoding structural and functional sequences, imperative for proper LV particle generation. Over the last decades, vector development has largely focused on the design of these plasmids. Firstly, only critical viral structural and functional sequences are provided and secondly, these sequences are divided over a certain number of individual plasmids either in cis (encoded by the LV) or trans (packaged as a protein within the LV particle), with a minimal overlap between viral sequences. This led to a LV production procedure where at least three different plasmids are used: (1) a packaging plasmid which provides all viral structural and enzymatic sequences (encoded by gag and pol) in trans to generate a functional particle, (2) a transfer plasmid providing the expression cassette in cis, cloned into the non-coding remains of the original lentiviral genome (Figure 1B, adapted from (Delenda 2004)) including a packaging signal and the two long terminal repeats (LTRs) of which the promoter activity has been deleted from the 3' LTR and (3) an envelope plasmid encoding an envelope glycoprotein (gp) consisting of a transmembranary domain (TM) and a receptorbinding domain (SU) that determines the LVs' tropism (Figure 1A).

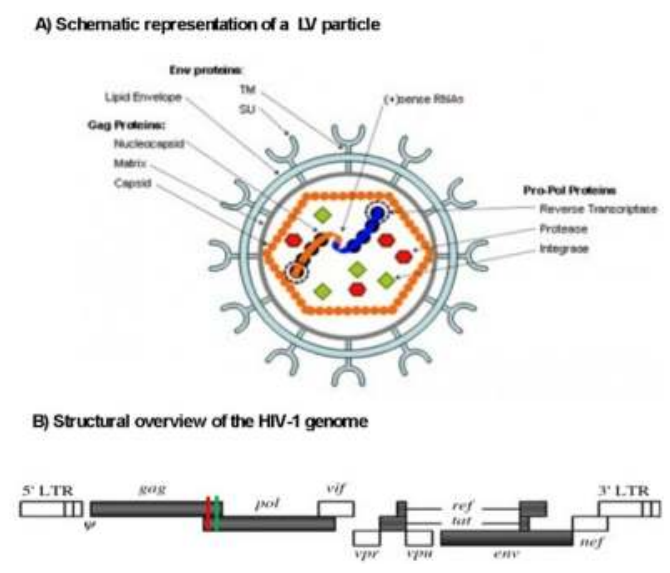

Figure 1. Schematic representation of an HIV-1 particle (A) and its genome (B). The diploid ssRNA genome of HIV-1 is stabilized by structural nucleocapsid proteins and together with the enzymatic proteins reverse transcriptase, protease and integrase packaged in a nucleocapsid structure, which in turn is enclosed by capsid proteins. This nucleocapsid is surrounded by a matrix protein layer and a producer cell derived phospholipid bilayer in which the envelope proteins consisting of an SU and TM part, are embedded (A). All proviral genes (gag, pol, pro, vif, vpr, vpu, ref, tat, env en nef) are flanked by two identical LTRs that consist of three regions: U3, R and U5. Within the U3 region, all proviral transcriptional control elements are situated such as the promoter and several enhancer sequences. $\Psi$ represents the packaging signal. At the $3^{\prime}$ end of the pol gene the central polypurine tract (red) and central termination sequence (green) are located. Both ensure the formation of a triple stranded DNA flap, crucial for nuclear entry of the pre-integration complex in non-dividing cells (B). 
Besides this division over different plasmids, other important construct optimization steps have been implemented. While in the first generation LV packaging plasmids the entire gag and pol genes were encoded together with all accessory regulatory and virulence genes, the second generation was multiply attenuated by removal of the four virulence genes, but not the regulatory genes tat and rev (Zufferey, Nagy et al. 1997). In the third generation, the rev gene is expressed from a separate plasmid and the tat gene is removed by insertion of a strong constitutive promoter replacing the U3 region in the $5^{\prime}$ LTR of the transfer plasmid (Dull, Zufferey et al. 1998). A major improvement was achieved with the development of SIN or self-inactivating LVs where a deletion in the U3 region of the 3' LTR of the transfer plasmid abolished the production of full-length vector RNA in transduced cells. This not only minimizes the risk for RCLs, but also reduces the chance that the viral LTR enhancers interfere with the expression cassette, which minimizes aberrant expression of adjacent cellular coding regions. Subsequently these and many other optimization steps paved the way towards a more effective and safer version of the lentiviral gene delivery vehicle (Romano, Claudio et al. 2003).

In addition to packaging and transfer plasmid optimization, also the envelope plasmid was modified by replacing the natural HIV-1 envelope gp with an alternative gp gene, most often the gp of vesicular stomatitis virus (VSV.G). This concept is called pseudotyping and VSV.G endowed the LV particle with an increased stability and broad cellular tropism (to most if not all mammalian cells). However, it became clear that for numerous in vivo applications, a broad tropism may not be desirable. First, the th that is encoded could be toxic to many cell types, e.g. pro-apoptotic or suicide genes, so a stringent control over the induction of tg expression in time and/or place is a necessity (Uch, Gerolami et al. 2003; Seo, Kim et al. 2009). A second point of concern is the risk for insertional mutagenesis; the more cells get infected, the higher this risk becomes. Although it has been demonstrated that LVs intrinsically exhibit low genotoxicity, clonal expansion and dominance of transduced hematopoietic progenitors have been reported in a clinical trial in which hematopoietic stem cells were genetically modified with a LV that expressed the $\beta$-globin gene for treatment of $\beta$-thalassemia (Fehse and Roeder 2008; Cavazzana-Calvo, Payen et al. 2010). Thirdly, while a broad tropism LV is favorable in antitumor immunotherapy to efficiently transduce antigen-presenting cells (APCs) which can induce an antigen specific immune response (Palmowski, Lopes et al. 2004), this is not desirable when a genetic disorder has to be restored as in this case the tg may not become an immunological target (Annoni, Battaglia et al. 2007). Finally, during production of pantropic viruses encoding oncogenes, narrow tropism vectors would be more valuable due to biosafety level handling requirements and safety issues (Barrilleaux and Knoepfler 2011). Therefore, in view of safety as well as applicability aspects, four main targeting strategies can be brought forward: targeted gene expression or transcriptional targeting, targeted gene translation or microRNA based (de)targeting, targeted infection or transductional targeting, and targeted integration of the proviral DNA. 


\section{Transcriptional targeting}

Most often a strong constitutive promoter with or without enhancer sequences is used to drive the LV encoded tg. These include the cytomegalovirus (CMV), spleen focus forming virus (SFFV), human polypeptide chain elongation factor-1alpha (EF-1alpha), phosphoglycerate kinase (PGK) and ubiquitin C promoters (Kim, Kim et al. 2007; Gilham, Lie et al. 2010; Li, Husic et al. 2010). Although these promoters generally induce strong and ubiquitous expression of the tg, they present some disadvantages. A first drawback is that they are more prone to promoter inactivation than cell-specific promoters. In addition, they are more potent in terms of activating the host-cell defense machinery and increasing the long-distance effects of insertional mutagenesis caused by their enhancer sequences (Liu, Wang et al. 2004; Stein, Ott et al. 2010; Singhal, Deng et al. 2011). These downsides resulted in the development of various strategies to allow cell-specific tg expression by incorporating cell type specific regulatory elements and/or promoter(s) in the expression cassette of the LV. Because of the availability of a large number of endogenous cellular promoters, targeted expression can be achieved to potentially any cell type or tissue. In addition, its advantage over unselective expression has been demonstrated in numerous studies (Di Nunzio, Maruggi et al. 2008; Kerns, Ryu et al. 2010; Cao, Sodhi et al. 2011). This is exemplified by a study where LV encoding iduronidase under the control of the hepatocyte specific albumin gene promoter was injected intravenously to treat mucopolysaccharidosis type I. While the same LV with a CMV promoter resulted in the induction of an immune response that diminished the tg expression over time, the albumin gene promoter enabled stable and prolonged tg expression with a partial correction of the pathology (Di Domenico, Di Napoli et al. 2006). In addition to hepatocyte specific targeting, an ever-growing list of cell-type specific promoters has been used for the specific expression in tissues such as the erythroid lineage, endothelial cells, myocardial cells, retinal cells, B cells, epidermal, hematopoietic stem cells, etcetera (Hanawa, Persons et al. 2002; De Palma, Venneri et al. 2003; Semple-Rowland, Eccles et al. 2007; Di Nunzio, Maruggi et al. 2008; Leuci, Gammaitoni et al. 2009; Kerns, Ryu et al. 2010; SempleRowland, Coggin et al. 2010; Cao, Sodhi et al. 2011; Lee, Fan et al. 2011; Friedrich, Nopora et al. 2012).

Besides the advantage of increased and prolonged expression levels when expressed in the target cell of choice, targeted expression can also be a necessity when the tg causes undesirable damage in non-target cells. For the treatment of Mpl-deficient aplastic anemia, for example, targeted transfer to hematopoietic stem cells is inevitable since ectopic $\mathrm{Mpl}$ expression causes lethal adverse reactions (Heckl, Wicke et al. 2011). The same holds true for toxin, proapoptotic or suicide gene encoding LVs used in anti-tumor therapy (Zheng, Chen et al. 2003; Hsieh, Chen et al. 2011). LVs are excellent candidates to modulate the tumor and its environment since they transduce both dividing cells such as most cancer cells but also non- or very slowly dividing cells such as cancer stem cells. Furthermore LVs are able to integrate in the genome of transduced cells, potentially generating clonal populations of genetically modified cancer cells, which may then spread throughout the tumor mass (Steffens, Tebbets et al. 2004). Vector targeting can be attempted by local vector delivery, although this raises practical concerns for non-solid and metastatic tumor cells. Consequently, systemic delivery of a 
targeted LVs and subsequent exclusive tg expression in cancer cells is the ultimate goal. Metastatic prostate cancer, for example, has been transcriptionally targeted in various ways (1) using a prostate-specific antigen (PSA) promoter to drive the expression of diphtheria toxin A, (2) using the prostate-stem cell antigen (PSCA) promoter to drive the expression of the Herpes Simplex Virus thymidine kinase (HSV-TK) suicide gene, or (3) combining the prostate-specific promoter ARR2PB and a short DNA sequence in the 5'-untranslated region that is recognized by the translation initiation factor eIF4E, often overexpressed in malignant cells, to drive the expression of the HSV-TK suicide gene (Yu, Chen et al. 2001; Zheng, Chen et al. 2003; Yu, Scott et al. 2006; Kimura, Koya et al. 2007; Petrigliano, Virk et al. 2009). Additionally, the tumor vasculature has been transcriptionally targeted using the endothelial specific Tie2 promoter to drive the conditionally toxic nitroreductase and subsequently diminish tumor growth (De Palma, Venneri et al. 2003). Another cancer cell type specific targeting strategy to limit tg expression to hepatocarcinoma was applied by Uch et al. They constructed a LV expressing HSV-TK under the control of the rat alpha-fetoprotein promoter elements (Uch, Gerolami et al. 2003). Besides cancer cell type specific strategies, also more generalized cancer targeting strategies have been developed. For example, as the human telomerase reverse transcriptase (hTERT) is expressed in most malignant tumors, its promoter has been used to drive the expression of the cytosine deaminase gene together with a green fluorescent protein (GFP) reporter gene. It was demonstrated that hTERT-positive tumors could be visualized after intratumoral injection of the LV in tumor-bearing nude mice and, more importantly, that significant tumor growth suppression was observed after delivery of the pro-drug 5-fluorocytosine ( $\mathrm{Yu}, \mathrm{Li}$ et al. 2011). Besides avoidance of toxic tg expression in a non-tumor cell, tumor specific gene therapy is also interesting for targeted imaging. For example, the use of the chimeric promoter EIIAPA containing the alpha-fetoprotein promoter and hepatitis B virus enhancer II was used to control the downstream expression of luciferase genes to subsequently assay the selective transcriptional activity by bioluminescence imaging (Hsieh, Chen et al. 2011).

As LVs efficiently infect non-dividing cells, they provide suitable platforms for tg delivery to multiple mammalian neuronal cell types. It has been shown that stereotactic injection of LVs in the brain parenchyma leads to transduction of the striatum, hippocampus and thalamus (Watson, Kobinger et al. 2002). Moreover, transcriptional targeting has proven to be a reliable technique to unravel the complexity of the nervous system by neuron and brain specific assessment of the effects of therapeutic proteins and RNA interference, or to investigate neuronal gene expression (Hioki, Kameda et al. 2007; Gascon, Paez-Gomez et al. 2008; Kuroda, Kutner et al. 2008; Peviani, Kurosaki et al. 2012). Regulatory sequences of rat neuron specific enolase, human glial fibrillary acidic protein and myelin basic protein have already been exploited to obtain LV-mediated selective gene targeting of neurons, astrocytes and oligodendrocytes, respectively (Jakobsson, Ericson et al. 2003; McIver, Lee et al. 2005). This has led to applications like subregional tg expression in the hippocampus using the hybrid hEF1alfa/HTLV promoter or neuron specific synapsin I promoter or targeting the central serotonergic neurons using a two-step transcriptional amplification strategy co-expressing the tryptophan hydroxylase-2 gene promoter with the chimeric enhancer GAL4/p65 (Kuroda, Kutner et al. 2008; Benzekhroufa, Liu et al. 2009). Next to the central nervous system, Bend- 
otti et al. recently focused on selective tg expression in mouse spinal cord motor neurons using motor neuron specific regulatory sequences derived from the promoter of the homeobox gene Hb9 (Benzekhroufa, Liu et al. 2009; Peviani, Kurosaki et al. 2012). However, neuron specific gene expression is not always very efficient and therefore several groups have attempted to improve the endogenous promoters using extra enhancers or artificial transcriptional activators such as the bidirectional promoter. For the latter, Liu et al. based their bidirectional promoter on the transcriptional activity of the human synapsin-1 promoter and the compact glial fibrillary acidic protein (GfaABC1D) promoter. In the opposite orientation, a minimal core promoter of 65 basepairs (bp) derived from the CMV promoter was joined upstream of both promoters, which were flanked with two gene expression cassettes. The 5' cassette transcribed the artificial transcriptional activator while the downstream cassette drove the expression of the gene of interest (Liu, Paton et al. 2008).

To fulfill the high expectations of gene therapy, both efficient delivery and sustained expression of the therapeutic gene are essential requirements. However, one of the major barriers to stable gene transfer by LVs is the development of innate and adaptive immune responses to the delivery vector and the transferred therapeutic tg. It became clear that in vivo administered broad tropism LVs efficiently transduce APCs and that these play a major role in the induction of tg specific immune responses (Annoni, Battaglia et al. 2007; Vandendriessche, Thorrez et al. 2007). Consequently transcriptional targeting can be applied to avoid tg expression in APCs. Brown et al. demonstrated stable GFP production by modified cells in vivo when tg expression was prevented in APCs (Brown, Venneri et al. 2006). Another study combined the hepatocyte specific enhanced transthyretin promoter with an APC-detargeting microRNA strategy, and showed the induction of GFP-specific regulatory $\mathrm{T}$ cells and the promotion of immunological tolerance (Annoni, Brown et al. 2009 ). Moreover, Matrai et al. demonstrated that hepatocyte-targeted expression by an integrase-defective LV (IDLV) induced tolerance to coagulation factor IX with prevention of the induction of neutralizing antibodies in mice (Matrai, Cantore et al. 2011). In contrast to gene therapy, immunotherapy pursuits the induction of a tg-specific immune response where APC-specific transduction is imperative. Therefore, LVs that drive tg expression via an APC-specific promoter have been developed. For instance Cui et al. used the HLA-DR promoter to target human MHC class $\mathrm{II}^{+}$cells like dendritic cells $\left(\mathrm{DCs}, \mathrm{CD} 83^{+}\right)$and macrophages $\left(\mathrm{CD} 14^{+}\right)$. They demonstrated the induction of an allogeneic T cell response in vitro (Cui, Golob et al. 2002). The dectin-2 promoter was used to target the expression of the human melanoma antigen NY-ESO-1 to murine APCs. After intravenous injection of the targeted LVs, selective tg expression in dectin- $2^{+}$splenic myeloid and plasmacytoid DCs as well as in $\mathrm{F} 4 / 80^{+}$macrophages was reported. Furthermore $\mathrm{CD} 11 \mathrm{c}^{+}$draining lymph node residing DCs were targeted after subcutaneous injection which resulted in strong NY-ESO- 1 specific $\mathrm{CD}^{+}$and $\mathrm{CD}^{+} \mathrm{T}$ cell responses (Lopes, Dewannieux et al. 2008). On the other hand, DC-induced tg specific tolerance has also been achieved after the use of a DC-specific promoter. When LVs carrying a CD11c promoter were used to make DC-specific transgenic mice by injecting the purified virus into the perivitelline space of single-cell embryos, the tg became an autologous antigen to 
which immunological tolerance was induced. Furthermore, this tg was only expressed in CD11 $\mathrm{c}^{+}$cells derived from the spleen, lymph nodes as well as the thymus (Zhang, Zou et al. 2009). Dresch et al. made use of the DC-STAMP promoter to engineer bone marrow-targeted LVs. Therefore, ex vivo transduced hematopoietic stem cells (HSC) were injected in lethally irradiated mice to make HSC chimeric animals (Dresch, Edelmann et al. 2008). When GFP expression was analyzed in the leukocyte population isolated from the spleen, the main DC subpopulations such as CD11b-CD8 ${ }^{+}$DCs, CD11b ${ }^{+}$CD8 ${ }^{-}$DCs, and plasmacytoid DCs were GFP positive next to a small percentage of $\mathrm{CD} 11 \mathrm{c}^{-} \mathrm{CD} 11 \mathrm{~b}^{+}$monocytes. Furthermore, tg expression could only be detected in $\mathrm{CD}_{11} \mathrm{c}^{+}$cells in the thymus. While the previous two tolerance inducing studies could be explained by the fact that undifferentiated DC precursors were transduced, Kimura et al. intravenously injected LVs encoding Trp2 driven by the MHCII promoter and also observed persistent tg expression selectively in the CD11c, $\mathrm{CD} 11 \mathrm{~b}$ and $\mathrm{CD}^{+} 9^{+} \mathrm{MHCII}^{+}$cells of the spleen without $\mathrm{CD}^{+} \mathrm{T}$ cell responses against $\mathrm{Trp} 2$ in contrast to a CMV carrying construct (Kimura, Koya et al. 2007; Dresch, Edelmann et al. 2008). The induction of tolerance in this study might be explained by the activation status of the transduced APCs. Induction of tg specific effector $\mathrm{T}$ cells requires fully activated APCs. Since, DC activation by LVs was shown to be dose-dependent, the LV titers used in these studies could explain the tolerogenic instead of stimulatory outcome (Breckpot, Emeagi et al. 2007; Breckpot, Escors et al. 2010).

Finally, also controllable or inducible tg expression can be a prerequisite. Reasons to use $\mathrm{tg}$ regulation are: to maintain appropriate levels of a gene product within the therapeutic range, to modulate, stop or resume tg expression in response to disease evolution, or in response to an endogenous molecule as e.g. the secretion of insulin induced by hyperglycemia. For human gene therapy, several ligand dependent transcription regulatory systems have been developed. For clinical applications, such systems need to be safe, specific, highly inducible, reversible and only show dose dependent activation with low basal activity while their ligands need to be bioavailable and low in immunogenicity (Toniatti, Bujard et al. 2004). One of the first and most widely used ligands is Tetracylin (Tet) or its more potent analog Doxycycline (Dox) (Efrat, Fusco-DeMane et al. 1995; Reiser, Lai et al. 2000). In contrast to the bacterial lac repressor/operator or the Cre-loxP recombinase system, it is applicable in vivo and reversible (Deuschle, Hipskind et al. 1990; Lakso, Sauer et al. 1992). The original bacterial Tet system is based on a Tet repressor protein (TetR) that inhibits the expression of the bacterial Tet resistance genes by binding to cognate operator sequences (TetO) in their regulatory regions. Upon the addition of Tet, the repressor is inactivated by allosteric change, allowing gene transcription (Gossen and Bujard 1992). The artificial Tet-off system is based on the generation of a hybrid transactivator (tTA) by fusion of the TetR to the transcription activation domain of the HSV VP16 protein. This fusion product will bind and activate transcription at promoters that include TetO while the presence of Dox impairs this binding, resulting in the shut off of gene expression (Furth, St Onge et al. 1994) (Figure 2A, adapted from (Ramezani and Hawley 2002). In contrast, the reverse Tet transactivator (rtTA), generated by ran- 
dom mutagenesis of tTA, requires Dox to bind to cognate operator sequences and activate transcription resulting in the inducible Tet-on system (Figure 2B).

\section{A) Tet-off system}

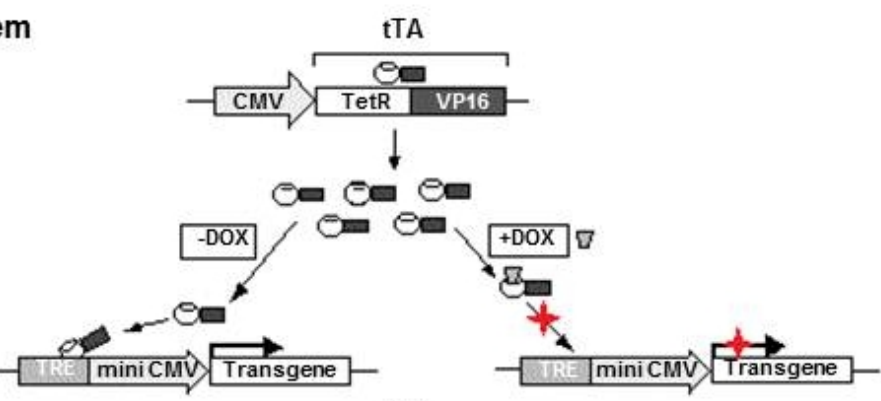

B) Tet-on system

rTTA

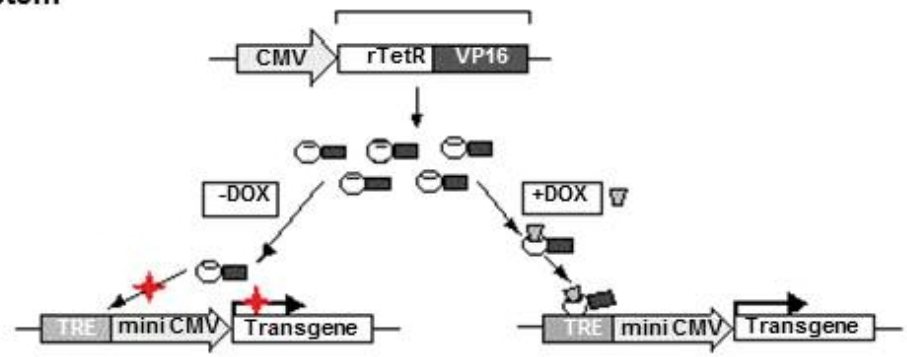

Figure 2. Representation of the artificial Tet-off (A) and Tet-on system (B). While the Dox binding transactivator (tTA) binds to the tetracycline-responsive promoter element (TRE) and stimulates tg transcription in the absence of $\operatorname{Dox}(\mathrm{A})$, the mutant reverse Tet-controlled transactivator (rtTA) binds to the TRE in the presence of Dox and stimulates transcription(B).

However, the in vivo applicability of the Tet system remained limited due to leakiness and insufficient induction levels. Therefore the Tet-on system has been optimized e.g. by isolating novel rtTA variants and incorporating a Dox-dependent trans-silencer called tTS which consists of the KRAB (Kruppel-Associated Box) trans-repressing domain of the human Kid-1 protein fused to the wild type TetR. This tTS has been used by the group of Szulc et al. to develop a LV-based conditional gene expression system for drug-controllable expression of inhibitory short hairpin RNAs (shRNAs), and reported on a robust and versatile system that governed the tight control over the tg expression both in vitro as well as in vivo among others to generate transgenic mice (Szulc, Wiznerowicz et al. 2006). Moreover, Dox is orally bioavailable, has a half-life of 14-22 hrs and has an excellent tissue penetration. Therefore numerous groups have used both the Tet-on and Tet-off system within LV-based gene reporter and therapeutic applications (Blomer, Naldini et al. 1997; Bahi, Boyer et al. 2004; Blesch, Conner et al. 2005; Liu, Wang et al. 2008; Hioki, Kuramoto et al. 2009; Adriani, Boyer et al. 2010). This is exemplified by a study of Seo et al. who developed an oncolytic LV- 
mediated Tet-on inducible system based on co-transduction of two LVs to drive the expression of a pro-apoptotic gene by the promoter of matrix-metalloproteinase-2 (MMP-2), which is highly expressed in several cancer cell lines. The first LV expressed a rtTA under the control of the MMP-2 promoter, whereas the second LV expressed the pro-apoptotic gene Bax, under the control of the tetracycline-responsive element (Seo, Kim et al. 2009). While most Dox inducible systems are based on the co-transduction of two LVs, all-in-one vectors have also been described recently (Ogueta, Yao et al. 2001; Barde, Zanta-Boussif et al. 2006; Herold, van den Brandt et al. 2008; Wiederschain, Wee et al. 2009; Benabdellah, Cobo et al. 2011). Furthermore, an extra Dox-regulated system based on the original TetR protein was developed in 1998. It serves as an alternative to the tTA- and rtTA-based systems because the latter were accompanied by secondary effects due to expression of the transactivator domains. Benabdellah et al. made use of the Dox-responsive cassette driving the expression of eGFP and the SFFV promoter expressing high amounts of the TetR protein in an all-in one vector system. This LV efficiently produced Dox-regulated cell lines, including primary human fibroblasts and human mesenchymal stem cells. However, a major concern using Dox remains the possibility to develop resistance to the antibioticum Tet, and although it seems a non-immunogenic system in several mouse strains, studies with intramuscularly delivered Tet-on activators in non-human primates did elicit a cellular and humoral response (LattaMahieu, Rolland et al. 2002).

Besides the Tet on/off systems, a plethora of inducible systems has been examined both in vitro and in vivo. An interesting strategy is based on the use of small molecules with distinct binding surfaces for two different polypeptides to modulate the activity of dimerizer-regulated systems. The prototype molecule is rapamycin, which mediates the heterodimer formation between two molecules (FK506-binding protein and FKBP rapamycin binding) that are coupled to a DNA binding domain (DBD) and transcription activation domain (AD) respectively (Pollock, Issner et al. 2000). The rapamycin inducible system has low basal activity because of the physical separation of the DBD and AD molecules, the ligand has a short half-life of about $4.5 \mathrm{hrs}$ although the induced gene expression lasts for days due to the strong stability of the DBD-AD assembled complex (Toniatti, Bujard et al. 2004). Tian et al. used a variant of this system to engineer LVs that produce a fusion protein between the furin-cleavable proinsulin and the self-dimerization mutant of FK506-binding protein to yield bioactive insulin in keratinocytes. Epidermal keratinocytes in culture, in stratified bioengineered epidermis as well as implanted in diabetic athymic mice released insulin within maximally $1 \mathrm{hr}$ after addition of rapamycin. Secretion slowed or stopped within 2-3 hrs after removal of the inducing agent. Even in diabetic animals with severe hyperglycemia, decreased serum glucose levels to normal levels were reported (Tian, Lei et al. 2008). The major disadvantage of this technique is the immunosuppressing activity of rapamycin and the only partial oral availability, which renders this system impractical for clinical applications.

Another strategy is based on the fact that heterologous proteins can be made hormone responsive by fusing them with the hormone-binding domain of steroid receptors. The bestcharacterized system is regulated by mifepristone or RU486, a synthetic progesterone

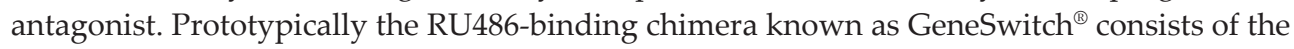


GAL4 DBD from Saccharomyces cerevisiae fused to the ligand-binding domain of a mutant progesterone receptor and the activation domain of the p65 subunit of human NF- $\kappa B$ (Abruzzese, Godin et al. 2000; Sirin and Park 2003). Upon ligand binding the GeneSwitch ${ }^{\circledR}$ protein binds to GAL4 upstream activating sequences in the promoter driving the expression of the tg of interest. An advantage of the GeneSwitch ${ }^{\circledR}$ system is that the majority of its components are modified human proteins with no impact on cell viability. Furthermore, usage of a mifepristone-inducible (auto-inducible) promoter to regulate expression of the chimeric transactivator dramatically reduced basal expression of the tg in the absence of the inducer, thereby improving the dynamic range of in vivo tg regulation (Shinoda, Hieda et al. 2009). In addition, although mifepristone has anti-progesterone and -glucocorticoid activities, the concentration needed for ligand-inducible transactivation of the target gene is much lower than the concentration producing an anti-progesterone effect in humans. However, it is thought that the lower dosage may still affect the ovarian cycle and exert a contraceptive activity. Therefore the search for other inducers that are unable to interact with endogenous progesterone would be more appropriate for clinical use (Sarkar 2002). As an alternative steroid-receptor based inducible system, the glucocorticosteroid responsive element (GRE5) was cloned into a LV (LV-GRE-IL10) encoding interleukin-10 (IL-10). Expression of IL-10 by LV-GRE-IL-10 appeared rapidly, was sustained and inducible in both ovine and human corneas in the presence of dexamethasone (Parker, Brereton et al. 2009). Another alternative can be the steroid hormone ecdysone, which plays a fundamental role during insect molting and metamorphosis. Ecdysteroids are considered safe because they are found in large amounts as phytoecdysteroids in vegetables, present in the human diet without detrimental effects. Mouse hematopoietic progenitors transduced with LVs containing an ecdysone-regulated GFP expression cassette efficiently turned GFP expression on and off in transplanted animals with low basal activity (Xu, Mizuguchi et al. 2003; Galimi, Saez et al. 2005). Possibly, several other systems will be developed to control tg expression after LV transduction. Potential systems could be based on the cell-cell communication quorum sensing process (Neddermann, Gargioli et al. 2003) or the naturally evolved mechanisms of antibiotic resistance to pristinamycin, a composite streptogramin antibiotic or erythromicin, a member of the macrolide antibiotics (Fussenegger, Morris et al. 2000; Roberts 2002).

\section{3. microRNA detargeting}

Recently, the concept of microRNA (miRNA) mediated post-transcriptional tg regulation was introduced in LV-based targeting. miRNAs are 21-22 nucleotide long non-coding fragments which are partially or extensively complementary to an endogenous mRNA molecule (Lai 2002). In mammals, over 400 different miRNAs have been identified so far, most of which are well conserved among species ranging from plants, worms, insects to humans (Brown and Naldini 2009). Some of these miRNAs are expressed ubiquitously whereas others are only expressed at certain developmental stages or in a certain cell type. Upon binding of a miRNA molecule to its complementary target sequence, repression of translation or direct destruction of the mRNA is induced. The detailed mechanisms involved in this post- 
transcriptional regulation process, do not lie within the scope of this book chapter but are reviewed elsewhere (Nelson, Kiriakidou et al. 2003; Bartel 2004). A brief description together with a schematic representation is depicted in Figure 3 (adapted from http://www.microrna.ic.cz/mirna4.html).

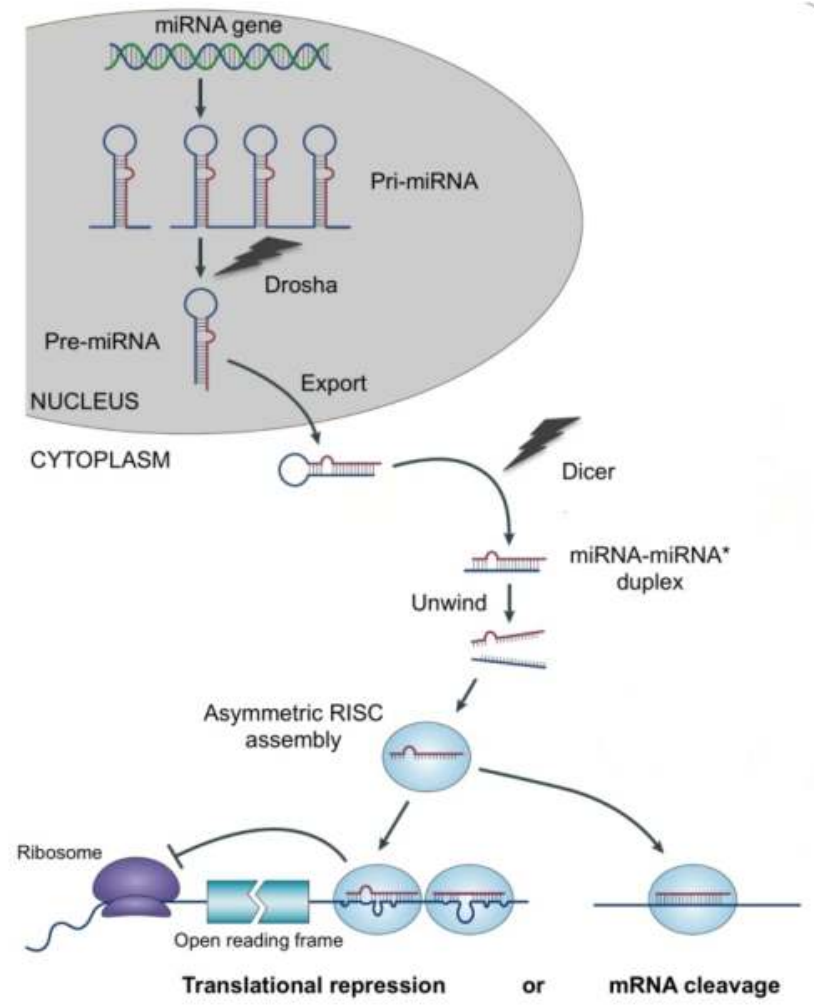

Figure 3. miRNA-based post-transcriptional gene silencing. Briefly, endogenous miRNA genes are transcribed by RNA polymerase II to pri-miRNA precursor molecules in the nucleus. These are processed to pre-miRNA by a specialized enzymatic pathway called Pasha/Drosha and will release the pre-miRNA in short hairpin RNA (shRNA). Then, these pre-miRNAs are exported to the cytoplasm where Dicer degrades most of the shRNA, leaving a miRNA duplex which is loaded onto the AGO complex (Argonaut), forming the preRISC (RNA Interference Silencing Complex). Subsequently the miRNA strand is degraded, leaving its complementary miRNA intact within the RISC complex. Then, this complex scans mRNAs and when complementation is found, the mRNA is degraded or the poly-A tail is removed, leading to mRNA destabilization or stalled mRNA translation.

In order to limit undesired vector tg expression, LVs encoding target sequences of endogenous miRNAs have been developed. By incorporating at the 3 'UTR region of the expression cassette one or more copies of a sequence that is perfectly complementary to a miRNA (miRNA tagging), the transgenic mRNA will be degraded or repressed in cells where the complementary miRNA is expressed. This new way of controlling tg expression at the level of the 
mRNA product came as a complementary strategy to transcriptional targeting since the latter is associated with some disadvantages such as: (1) difficulty to identify and faithfully reconstitute a gene's promoter; (2) for integrating LVs, promoters and enhancers can be trapped, leading to aberrant expression (De Palma, Montini et al. 2005), (3) transcription can be promiscuous and (4) only few genes have truly cell-specific transcriptional patterns while several promoters are active in many different cell types or states. Moreover, as miRNAs regulate expression at the post-transcriptional level, copy number and vector integration site have no appreciable effect on their regulation, which ensures consistent control throughout the transduced cell population.

Successful outcomes of LV-based gene therapy have long been precluded by the development of tg-specific immunity as a consequence of the direct expression of the tg product by professional APCs. Therefore Brown et al. challenged mice with LVs encoding a target sequence for miRNA-142-3p, a microRNA specifically expressed in the hematopoietic lineages. Upon injection, they demonstrated a 100-fold suppression of reporter gene expression in intravascular and extravascular hematopoietic lineages, including APCs (Brown, Venneri et al. 2006). One year later, its usefulness was evidenced by the miRNA-142-3p regulated LV mediated stable correction of hemophilia B in mice (Brown, Cantore et al. 2007). Its expression leads to reduced tg expression in APCs and subsequently lower anti-tg immune responses. Moreover it was demonstrated that in vivo delivery of this post-transcriptionally regulated LV induced tg-specific Foxp $3^{+}$regulatory $\mathrm{CD}^{+} \mathrm{T}$ cells, which promoted immunologic tolerance (Annoni, Brown et al. 2009 ). Curiously, they also reported the necessity of a hepatocyte specific promoter for this immunological tolerance. So, these studies showed the impressive potential of miRNA-based detargeting to overcome a major hurdle for clinical gene therapy, however also other factors than tg expression in APCs seem to influence the immunological outcome of a gene transfer procedure. Examples are the type of vector used, the tissue targeted and the presence of inflammation (Brown and Lillicrap 2002; Cao, FurlanFreguia et al. 2007).

Another reason to pursue stringent tg regulation, is to express the tg in a specific developmental state. Brown et al. showed that multiple endogenous miRNAs can be used to achieve tg expression patterns that rapidly adjust and sharply discriminate among the myeloid and lymphoid lineage in therapeutically relevant HSCs and their progeny with miRNA-223, or among immature and mature APCs using miRNA-155 (Brown, Gentner et al. 2007). Another example is provided by Gentner et al. who used the miRNA-126 target sequence to detarget $\mathrm{tg}$ expression from stem cells and progenitors from the hematopoietic cell lineage in order to avoid expression of the highly toxic GALC in these stages, while inducing GALC expression in mature cells from the hematopoietic lineage to correct globoid cell leukodystrophy (Gentner, Visigalli et al. 2010). Furthermore the group of Sachdeva et al. used miRNA-292 regulated LVs to visualize and segregate differentiating neural progenitors in pluripotent cultures and demonstrated that miRNA-regulated vectors allow a potentially broad use on stem cell applications (Sachdeva, Jonsson et al. 2010). Finally, Sadelain et al. used LVs that encode antigen specific receptors together with target sites for miRNA-181a to suppress the expression of the receptor in late thymocytes. This avoided clonal deletion of antigen specific $\mathrm{T}$ cells in 
the thymus and subsequent challenge with antigen expressing tumors did not result in tumor growth (Papapetrou, Kovalovsky et al. 2009).

Furthermore this technology is useful as a mechanism to increase vector safety and efficacy by limiting the expression of a toxic or pro-apoptotic tg to certain target cells. For example Lachmann et al. used the miRNA-150 target sequence to suppress GFP expression in lymphocytes and thereby prevented tg-induced lymphotoxicity (Lachmann, Jagielska et al. 2011). On the other hand unrestrained growth of transduced cells could also be avoided using miRNA-based detargeting when growth-promoting gens are replaced (Hawley, Fong et al. 1998). Moreover, miRNA-based regulation could be desirable when targeted gene expression is needed to assess the contribution of a particular cell type to physiological processes or for the development of new therapeutic strategies. This is exemplified by the work of Colin et al. who segregated tg expression between neurons and astrocytes following injection into the brain by exploiting the activity of miRNA-124 (Colin, Faideau et al. 2009). Another miRNA-based targeting strategy developed a few years ago was the concept of miRNA sponges, decoys, erasers, antagomirs or knockdowns (Ebert, Neilson et al. 2007; Scherr, Venturini et al. 2007; Gentner, Schira et al. 2009). Therefore vectors expressing miRNA target sites can effectively saturate an endogenous miRNA and prevent it from regulating its natural targets. This technology enables a new way of investigating miRNA biology and has already been used to study the role of miRNAs in cancer, cardiac function and hematopoiesis (Scherr, Venturini et al. 2007; Bonci, Coppola et al. 2008; Kumar, Erkeland et al. 2008; Sayed, Rane et al. 2008; Gentner, Schira et al. 2009; Valastyan, Reinhardt et al. 2009).

A possible concern of miRNA-based detargeting is whether sufficient target knockdown can be achieved for specific applications without escape mutants arising (Kelly, Hadac et al. 2008). In addition, it is highly likely that overexpression of the synthetic target sites will saturate their corresponding endogenous miRNAs and deregulate expression of natural targets with deleterious consequences. However, the latter has not been reported so far (Brown, Gentner et al. 2007). Moreover, miRNA-based regulation is a very robust system since at low copy vector number miRNA regulation of tg expression remains effective. Apparently, when a threshold miRNA concentration is present, the tg will be suppressed. This robustness can probably be explained by the perfect complementarity of the target sequence and the endogenous miRNA sequence. Indeed, when imperfectly complementary sites were used, this did result in a detectable decrease in target suppression, although only at very high vector copy numbers. So, although it should be recognized that the knowledge regarding miRNA biology and function is still limited, this strategy holds great potential to carefully move towards clinical translation (Brown and Naldini 2009)

\section{Transductional targeting}

Although the strategies described above demonstrate cell-specific gene expression, they often require broad tropism LVs which does not reduce the risk for RCL formation or insertional mutagenesis. Therefore transductional targeting of LVs seems a more interesting 
strategy to tackle both safety and efficacy concerns. The concept of swapping the viral envelope proteins of different viral species is called pseudotyping. Already in 1979, the envelope glycoprotein of the avian retrovirus was used to pseudotype VSV virions in order to selectively enrich for VSV temperature-sensitive mutants of VSV.G biosynthesis (Lodish and Weiss 1979). Later it was shown that wild type HIV-1 particles which were produced in cells that were infected with another virus, e.g. murine leukemia virus (MLV) or VSV, led to the generation of phenotypically mixed virions with an expanded host range (Canivet, Hoffman et al. 1990; Zhu, Chen et al. 1990). These observations introduced the concept of pseudotyping and in the early 90's the gp160 sequence of a replication defective HIV-1 derived LV was replaced by a MLV gp (Page, Landau et al. 1990). Later on the natural envelope gp from an MLV-based vector was replaced with the viral attachment protein of VSV (Emi, Friedmann et al. 1991; Burns, Friedmann et al. 1993). Today, most synthetic LVs are pseudotyped with a heterologous envelope protein to increase their stability, infectivity and safety. Notably, the first LVs were not pseudotyped but displayed the native HIV-1 envelope protein at their surface. This limited their tropism to CD4-expressing cells (Dropulic 2011). Interestingly, VSV.G pseudotyped vectors are more stable than their natural counterparts. This allows concentration to higher titers by ultracentrifugation and confers broad tropism, as VSV.G binds to a still unknown ubiquitous membrane component (Cronin, Zhang et al. 2005). This superior transduction efficiency comes in handy for the treatment of genetic disorders such as $\beta$-thalassemia and X-linked adenoleukodystrophy (Cartier, Hacein-Bey-Abina et al. 2009; Cavazzana-Calvo, Payen et al. 2010). Nonetheless, VSV.G pseudotyped LVs also present several downsides. Firstly, the VSV gp is cytotoxic when expressed constitutively at high concentrations, which impedes the production of stable packaging cell lines (Lopes, Dewannieux et al. 2011). In addition, cytotoxicity associated with VSV.G pseudotyped LVs has been observed when in vivo administered at high concentration, in comparison with other pseudotypes (Watson, Kobinger et al. 2002). Another critical hurdle for systemic delivery using VSV.G pseudotyped LVs is their susceptibility to neutralization by human serum complement, although this can be bypassed by polyethylene glycol-modification (PEGylation) of the virions (DePolo, Reed et al. 2000; Croyle, Callahan et al. 2004).

An ever-growing list of alternative pantropic as well as ecotropic naturally occurring gps has been evaluated for LV pseudotyping. These vary in origin, tropism, titer, stability, efficiency of packaging, inactivation by complement, efficiency of cell transduction and induction of an immune response (Cronin, Zhang et al. 2005). They can be of retroviral origin such as those from T-lymphotropic virus, maedi-visna virus, MLV, feline endogenous retrovirus and gibbon ape leukemia virus (GALV) (Rasko, Battini et al. 1999; Stitz, Buchholz et al. 2000; Zeilfelder and Bosch 2001; Strang, Ikeda et al. 2004; Sakuma, De Ravin et al. 2010). In general, LVs pseudotyped with a $\gamma$-retroviral envelope transduce CD $34^{+}$hematopoetic precursors, a property that has been used for the correction of X-linked severe combined immunodeficiency (SCID) using the GALV or MLV-A envelopes (Cavazzana-Calvo, Hacein-Bey et al. 2000; Gaspar, Parsley et al. 2004). Nonetheless, envelope gps of numerous non-retroviral families have been used as well to pseudotype LVs. A first example is provided by the Togaviridae family, where their envelope gps (from alphaviruses such as the Ross River virus) equips the LV with a mouse and human DC-specific tropism when injected intravenously 
(Strang, Takeuchi et al. 2005), and with an astrocyte and oligodendrocyte specific tropism when injected into the mouse brain (Kang, Stein et al. 2002). Another example is provided by the family of the Baculoviridae where the gp64 gp ensures high particle stability in addition to a hepatocyte specific tropism (Matsui, Hegadorn et al. 2011). LVs pseudotyped with the lymphocytic choriomeningitis virus (LCMV) envelope from the Arenaviridae preferentially transduce cells from the central nervous system such as neural stem cells and progenitor cells, and also to glioma cells and insulin secreting $\beta$-cells (Kobinger, Deng et al. 2004; Miletic, Fischer et al. 2004; Stein, Martins et al. 2005). As there is an increasing interest in the development of gene therapeutic strategies for malignant gliomas, the most frequent primary brain tumors with very poor prognosis, several groups report on the use of LCMV gp pseudotyped LVs to target almost exclusively astrocytes, the main source of malignant glioma cells (Beyer, Westphal et al. 2002; Miletic, Fischer et al. 2004; Steffens, Tebbets et al. 2004). The $\mathrm{H}$ and $\mathrm{F}$ envelope proteins from the Paramyxoviridae family, such as those derived from measles viruses, provide LVs with the capacity to bind to SLAM and CD46, which confers efficient virus entry, nuclear transport and integration in non-activated B and T lymphocytes. This property is particularly important, since primary unstimulated B and T cells are generally difficult to transduce if not pre-treated to induce progression through the cell cycle (e.g. through stimulation with anti-CD3/anti-CD28 antibodies or cytokines) (Frecha, Levy et al. 2010; Frecha, Levy et al. 2011). To transduce airway epithelial cells efficiently, envelope proteins from several viruses that infect respiratory tissues or cells have been evaluated. For efficient transduction of unconditioned airway epithelial cells from the apical side, envelopes derived from the ebola virus (Filoviridae), members of the Paramyxoviridae such as the respiratory syncytial (RSV) and sendai viruses, and members of the Orthomyxoviridae such as the influenza and fowl plaque viruses have been evaluated (Kobinger, Weiner et al. 2001; Nefkens, Garcia et al. 2007; Mitomo, Griesenbach et al. 2010). Surprisingly, it has been reported that the $S$ protein of the severe acute respiratory syndrome-associated coronavirus (Coronaviridae) mediates entry into hepatoma cell lines (Hofmann, Hattermann et al. 2004). Finally, although the vesicular stomatitis, mokola and rabies virus are all derived from the Rhabdoviridae family, only LVs pseudotyped with the rabies-G envelope enable retrograde transport to motoneurons of the spinal cord upon intramuscular injection or to the thalamus upon striatal injection. In contrast, VSV.G displaying LVs transduce cells only locally while mokola-pseudotyped LVs preferentially target non-neuronal glial cells (Mazarakis, Azzouz et al. 2001; Azzouz, Ralph et al. 2004; Wong, Azzouz et al. 2004; Colin, Faideau et al. 2009; Calame, Cachafeiro et al. 2011).

Although the use of an existing viral envelope gp seems the most straightforward way to pseudotype LVs, a natural variant with the desired delivery properties is not available for every therapeutic application. Moreover, natural gps can come with limitations such as sensitivity to neutralization by the host immune response, lack of specificity and/or insufficient transduction efficiency. Also their production and purification can be inefficient (Schaffer, Koerber et al. 2008). Therefore, the development of LVs with customized, user-defined gene delivery properties by molecular engineering of the envelope gps is an alternative strategy to retarget the LV to specific cell-surface receptors. This molecular engineering has become a collective term for many different strategies, which will be described below. 
A first strategy to alter the tropism of a virally derived gp is by rational point and domain mutagenesis. This is exemplified by the DC-specific targeting strategy from Yang et al. Certain subsets of DCs carry the DC-SIGN protein (also known as CD209) on their surface, which is a C-type lectin-like receptor that potentiates rapid binding and endocytosis of materials. The sindbis virus envelope gp consists of two integral membrane gps that form a heterodimer and function as one unit. The fusogenic monomer is E1 and needs binding via E2 to mediate low $\mathrm{pH}$-dependent fusion. The latter binds to the DC-SIGN receptor, next to the canonical viral receptor heparin sulfate, expressed by many cell types. Since both protein binding sites are physically separated, selective mutation at the E2 monomer is possible, abrogating the heparin sulfate binding part while leaving the DC-SIGN binding part intact. By pseudotyping a LV with this mutated sindbis virus derived envelope gp, targeted infection of DCs in vivo after direct subcutaneous administration was achieved. Moreover, this elicited a strong antigen-specific immune response (Yang, Yang et al. 2008; Hu, Dai et al. 2010). Another example is the substitution of the V3-loop region of the SIV envelope gp with the corresponding region of a T cell tropic HIV-1 to create a T-cell targeted MLV vector, pseudotyped with this engineered SIV gp (Steidl, Stitz et al. 2002). A final example is provided by Dylla et al. who diminished the alfa-dystroglycan affinity of the LCMV WE45 strain envelope gp by a point mutation. When a FIV derived LV was pseudotyped with this point mutated LCMV gp, their intravenous injection in adult mice yielded low transduction efficiencies in hepatocytes in contrast to abundant liver and cardiomyocyte transduction with the wild type LCMV gp pseudotyped FIVs (Dylla, Xie et al. 2011).

Apart from genetic alterations, chemical modifications can also alter LV tropism. PEGylation of VSV.G pseudotyped LVs is one such example where the LVs' tropism is not altered. Nevertheless, chemical modifications can lead to targeted gene delivery vehicles, for example by tagging the MLV vector with galactose to selectively transduce human hepatoma cell lines expressing asialo-gp receptors specific for oligosaccharides with terminal galactose residues (Neda, Wu et al. 1991). Furthermore, Morizono et al. reported the production of LVs pseudotyped with sindbis virus gps in the presence of deoxymannojirimycin. This modification altered the structures of N-glycans from complex to high mannose structures as it inhibits mannosidase. This led to DC-SIGN specific binding although the gps were genetically modified to prevent interaction with DC-SIGN (Morizono, Ku et al. 2010). Furthermore it was demonstrated that binding of sindbis virus gp to DCs is directly related to the amount of high-mannose structures on the gp (Tai, Froelich et al. 2011). Unfortunately, the effectiveness of the chemically modified particles strongly depends on the reaction conditions of the applied modifications.

Other chimeric envelope gps can be generated by covalently fusing a short peptide, a ligand or an antibody to an envelope gp. The advantages of short peptides are that they don't severely disrupt the original envelope gps' function and that via high-throughput library approaches, targeted peptides with strong binding affinity and unlimited specificity within the context of a particular gp can be generated (Schaffer, Koerber et al. 2008). However, they can hinder multimerisation of capsid monomers, create fusion products with lower thermostability and hinder proper intracellular trafficking of the gp during viral production. The latter 
is exemplified by the blockage in trafficking in the producer cells to the plasma membrane of VSV.G when linked to a collagen-binding motif (Guibinga, Hall et al. 2004). Different kinds of ligands such as cytokines and growth factors have been linked to the amino-terminal region or receptor-binding domain of the envelope gp, most often derived of MLV. This is amongst others exemplified by fusion of the MLV gp to hepatocyte growth factor to target the LV to hepatocytes (Nguyen, Pages et al. 1998), or to the insulin-like growth factor (IGF-I) (Chadwick, Morling et al. 1999). Interestingly, these ligands can elevate the transduction efficiency by altering the targets' physiological state. When the fusion product of the MLV gp and IL-2 is used to pseudotype LVs, a 34-fold higher infection efficiency was observed of quiescent IL-2 receptor expressing cells compared to LVs pseudotyped with the wild type MLV gp. This was explained by IL-2 induced activation of the cell cycle from the otherwise barely transducible quiescent cells (Maurice, Mazur et al. 1999). However, a very low to unobservable transduction profile is often reported which can be attributed to sequestration of the LV particles at the target cell surface, directing the viral particle to a degradation pathway after endocytosis and/or inability of the fusion product to trigger a conformational change essential for viral fusion and subsequent infection (Lavillette, Russell et al. 2001; Katane, Takao et al. 2002). In addition to peptides and ligands, also antibodies and their derivatives can be used. In general, single chain variable fragments or scFvs offer higher specificity than short peptides but as they are larger in size, the chance that they disrupt the process of conformational changes of the gp to mediate membrane fusion increases. Therefore scFvs are most often linked to a spacer peptide that permits proper conformation of both the scFv domain and the envelope gp as exemplified by the fusion of the MLV gp to a scFv against MHC class I (Karavanas, Marin et al. 2002). For LV targeting to APCs, several attempts have been made to couple an anti-MHC class II scFv to an ecotropic gp such as MLV or VSV.G (Dreja and Piechaczyk 2006; Gennari, Lopes et al. 2009). Recently, the use of DARPins or designed ankyrin repeat proteins has been reported. These can be fused to the H protein of measles virus for example and then be co-displayed with the fusogenic F protein on the surface of the LV. The advantage is that DARPins can be selected to become high-affinity binders to any kind of target molecule thus this seems a promising alternative to scFvs for retargeting LVs (Munch, Muhlebach et al. 2011). So, in general, the use of chimeric envelope proteins for LV targeting has proven to offer tremendous opportunities but at the same time to be a challenge as the function of chimeric gps is often severely compromised which leads to a very inefficient transduction profile (Fielding, Maurice et al. 1998; Dreja and Piechaczyk 2006; Waehler, Russell et al. 2007; Buchholz, Duerner et al. 2008).

Several solutions have been created to circumvent the problems associated with the formation of conformational dysfunctional fusion products. One solution is the inclusion of a protease cleavable peptide between the gp and the ligand. This is certainly an interesting strategy for the targeting of tumor cells, as they secrete MMP, which degrade the extracellular matrix to metastasize. By linking a proline-rich hinge and an MMP cleavage site to the fusion product of a scFv recognizing carcinoembryonic antigen (CEA) and the MLV gp, selective targeting of CEA-positive cells after in vivo injection of producer cells at the tumor site was observed (Chowdhury, Chester et al. 2004). Taking this hinge region idea one step further, the concept of 'molecular bridges' was introduced where a bispecific linker mole- 
cule recognizes both the viral gp as well as the molecular determinant on the target cell. This concept is based on a bridging system that was introduced more than 20 years ago and where three different linker molecules were involved: two biotinylated antibodies that bound the MLV gp and MHC class I or II proteins on the target cells respectively, and a bridging streptavidin molecule linking both antibodies. This led to the generation of a MLV that was capable of transducing MHC class I and II expressing cells (Roux, Jeanteur et al. 1989). Subsequently, two-protein molecular bridges have been exploited based on the avidin-biotin system. A recent example is provided by O'Leary et al. who used a detoxified recombinant form of the full-length botulinum neurotoxin, fused to core streptavidin that for its part was coupled to a biotinylated LV. This envelope gp construct endowed the LV particle with considerable neuron selectivity in vitro as well as in vivo after injection into the trachea (O'Leary, Ovsepian et al. 2011). Nowadays, alternative linkers such as ligand-receptor, chemical conjugations and monoclonal antibodies have been exploited to retarget LVs as well (Roux, Jeanteur et al. 1989; Boerger, Snitkovsky et al. 1999). For the latter, the E2 protein of the sindbis gp has been modified to contain the Fc-binding domain (ZZ domain) of protein A, making it possible to bind to a monoclonal antibody specific for a target molecule via its Fab antigen recognition end (Morizono, Xie et al. 2005). However, doubts are raised about the affinity of the adaptor-virus complex, as this may not be sufficient to prevent dissociation within the patient's blood. Moreover, complexity ascends as both the virion as the adaptor must be produced, purified and fully characterized for clinical approval. Another alternative possibility is to co-display a chimeric envelope gp together with a wild type gp such as VSV.G to enhance the transduction efficiency (Maurice, Verhoeyen et al. 2002; Verhoeyen, Dardalhon et al. 2003; Verhoeyen, Wiznerowicz et al. 2005). However, this had also limited success due to partial loss of targeting specificity. Therefore, a final alternative is the usage of a mutated fusogenic but binding-defective envelope gp to mediate fusion upon binding by the chimeric gp. The group of Lin et al. co-expressed the MLV gp fused to soluble Fms-like tyrosine kinase 3 (Flt3)-ligand together with a binding-defective influenza hemagglutinin protein from the fowl plague virus rostock 34 (HAmu). When LVs were pseudotyped with both of these gps, Flt3-targeted transduction was enhanced when compared to LVs without HAmu and could be competed away by the addition of soluble Flt3ligand (Lin, Kasahara et al. 2001). Another more straightforward strategy is the use of the $\mathrm{E} 1 / \mathrm{E} 2$ heterodimer gp of sindbis virus as the fusion and binding functions are already separated over two different monomers. By mutating the binding E2 monomer, its binding property can be completely abolished. Therefore, this binding defective E2 protein forms an ideal scaffold for cell-specific antibody conjugation to confer specific tropism to an endless list of cell types such as P-gp-expressing melanoma progenitor cells and endothelial cells (Morizono, Xie et al. 2005; Pariente, Mao et al. 2008). A drawback is that they only induce fusion upon low $\mathrm{pH}$. Therefore alternatives were explored such as the $\mathrm{H}$ and $\mathrm{F}$ protein of the measles virus, which induce fusion without the need for endocytosis (Earp, Delos et al. 2005; Funke, Schneider et al. 2009). This is exemplified by a study were a binding defective form of the $\mathrm{H}$ protein was fused to a CD20 specific scFv to pseudotype LVs. When these LVs were used to kill cells in culture, they selectively killed the CD20+ human lymphocytes in co-culture with CD20- cells. This demonstrated the ability of these LVs to exclusively transfer a po- 
tentially hazardous therapeutic protein into targeted cell populations with virtual absence of background transduction in non-target cells (Funke, Maisner et al. 2008). Meanwhile, a broad variety of surface antigens has been successfully targeted using this strategy (Blechacz and Russell 2008)

A fourth strategy to target LVs is based on two concepts: (1) the separation of binding and fusion functions over two distinct envelope molecules and (2) the ability of LVs to incorporate host cell proteins into their envelope as they bud from the plasmamembrane of their producer cells (Chandrashekran, Gordon et al. 2004; Kueng, Leb et al. 2007). Chandrashekran et al. reported on efficient and specific targeting to human cells expressing stem cell factor (SCF) receptor (c-kit) by an ecotropic gp pseudotyped LV which also displayed surface $\mathrm{SCF}$. Another example is the overexpression of the HIV-1 derived primary receptor CD4 and fusogenic co-receptor CXCR4 or CCR5 on the membrane of producer cells. From these cells, LVs were generated that infect HIV-1 envelope gp expressing cells next to cells infected with HIV-1, enabling the development of novel antiviral therapy approaches (Somia, Miyoshi et al. 2000). Since the transduction efficiency was relatively low, LV co-enveloped with the HIV-1 cellular receptor CD4 and the E2 protein from sindbis virus were created. These turned out to have a higher infectivity level than in the former strategy (Lee, Dang et al. 2011). In another study the binding defective but fusogenic E1/E2 heterodimer was used to be co-displayed with a separate membrane bound anti-CD20 antibody in order to transduce exclusively CD20+ B cells (Lei, Joo et al. 2009). Today, numerous examples are found that apply this principle to target the following: immunoglobulin-expressing $\mathrm{B}$ cells, $\mathrm{CD}^{+} \mathrm{T}$ cells and CD117 $7^{+}$HSCs (Ziegler, Yang et al. 2008; Froelich, Ziegler et al. 2009; Yang, Joo et al. 2009). However, clinical applications with LVs displaying scFvs are hampered by lack of stability, size and immunogenicity leading to the development of neutralizing antibodies. To solve these problems, we developed the Nanobody $(\mathrm{Nb})$ display technology (Goyvaerts, De Groeve et al. 2012). In this strategy, a fusogenic but binding-defective form of VSV.G (VSV.GS) (Zhang, Kutner et al. 2010) is co-displayed with a surface bound form of a cell-specific $\mathrm{Nb}$ to confer target binding (Figure 4). Some twenty years ago, Hamers-Casterman et al. discovered that part of the humoral response of Camelids is based on a unique repertoire of antibodies, which only consisted of two heavy chains (Hamers-Casterman, Atarhouch et al. 1993). The antigen binding part of these antibodies is composed of only one single variable region, termed $\mathrm{VHH}$ or $\mathrm{Nb}$. These $\mathrm{Nbs}$ have unique characteristics and offer many advantages over scFvs to target LVs to specific cell types. These include (1) they are highly soluble, (2) they can refold after denaturation whilst retaining their binding capacity, (3) cloning and selection of antigen-specific Nbs obviate the need for construction and screening of large libraries, (4) as Nbs can be fused to other proteins, it is possible to present them on the cell membrane of a producer cell line, thus generating LVs that incorporate a cell-specific $\mathrm{Nb}$ in their envelope during budding. Using the $\mathrm{Nb}$ display technology, we demonstrated production of stable $\mathrm{Nb}$ pseudotyped LV stocks at high titers with a DC subtype specific transduction profile both in vitro as well as in vivo (Goyvaerts, De Groeve et al. 2012). As ligand specific Nbs can be generated to potentially every cell surface molecule, this technology will be applicable to target LVs to every cell type for which cell specific surface molecules are characterized (Gainkam, Huang et al. 2008; Vaneycken, Devoogdt et al. 2011). 
The downside of the use of the above-described strategies is that they rely on the fusogenic capacity of a gp that is derived from viruses infectious to humans such as VSV, measles virus, sindbis virus and MLV. Their exposure to the complement or immune system, leading to anti-gp antibodies, might limit their clinical applicability. To surmount these obstacles, Frecha et al. pseudotyped LVs with a mutant fusogenic gp derived from an endogenous feline virus, named RD114. The mutant RD114 gp is an attractive candidate for in vivo use as it is resistant to degradation by the human complement. By co-displaying the early-acting-cytokine SCF together with mutant RD114 gp, human CD34 ${ }^{+}$HSCs could be targeted in vivo (Frecha, Fusil et al. 2011; Frecha, Costa et al. 2012). SCF was responsible for a slight and transient stimulation of the HSCs while preserving the 'stemness' of the targeted HSCs. In that way, the need for CD3/CD28 or cytokine pretreatment was obviated. Springfeld et al. recently pseudotyped LVs with the $\mathrm{H}$ and F gps of the Tupaia paramyxovirus (TPMV), an animal virus without close human pathogenic relatives. Moreover, as this virus does not infect human cells, detargeting the $\mathrm{H}$ protein from its natural receptors is unnecessary. When LVs were pseudotyped with the TPMV envelope protein linked to an anti-CD20 single chain antibody, selective transduction of $\mathrm{CD}_{2} 0^{+}$cells, including quiescent primary human B cells, was reported (Enkirch, Kneissl et al. 2012).

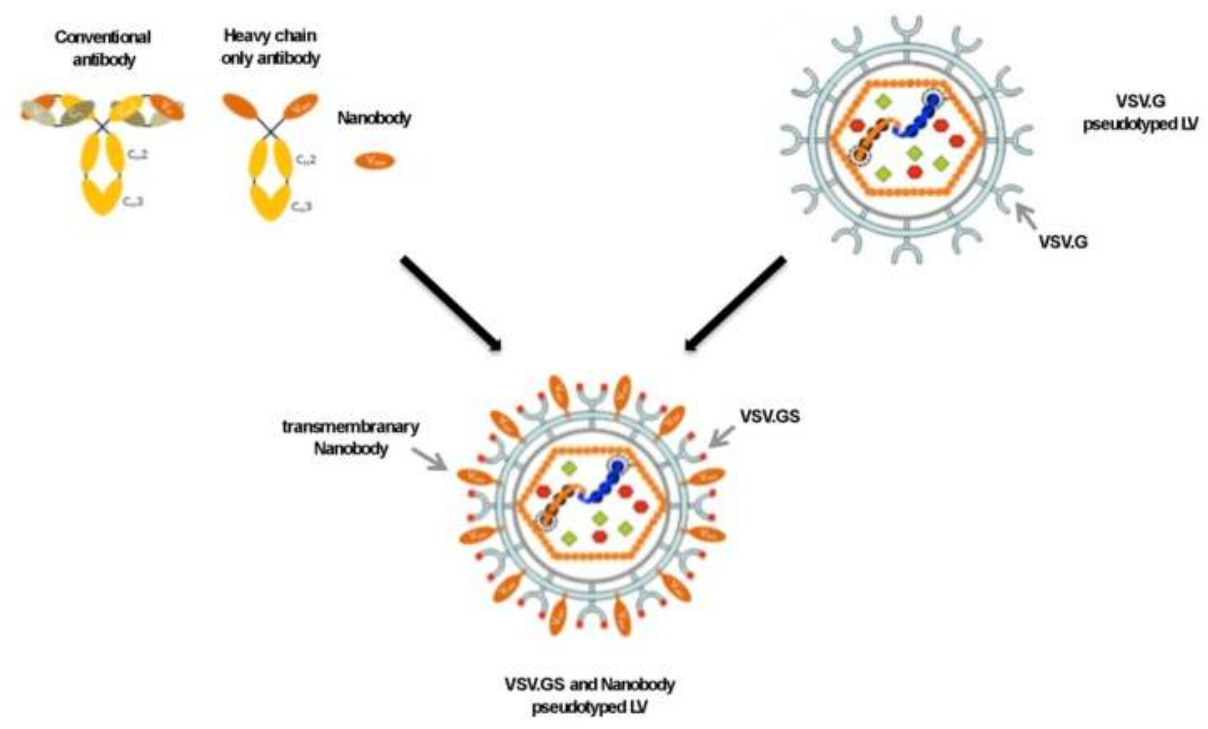

Figure 4. Principle of the Nb display technology. The Nb display technology is based on the fact that LVs need to bind and fuse with the membrane of a target cell for proper infection. While VSV.G accounts for both of these functions, we propose to separate these functions over two different molecules: (1) binding via a membrane bound $\mathrm{Nb}$ against the target cell of choice and (2) fusion via VSV.GS, which is a binding defective truncated version of VSV.G.

Recently an innovative alternative strategy has been described by Mannell et al. for site specific vascular gene delivery. In this case, the LVs were first coupled to magnetic nanoparti- 
cles, which were in turn coupled to lipid microbubbles. LVs coupled to magnetic nanoparticles to target them to specific cell types in vitro using an external magnetic field has been described before. However, when these LV-nanoparticle constructs are considered for in vivo use, a sufficient magnetic moment is needed as the particles are subject to flow velocity within the blood vessels. As the magnetic moment is proportional to particle size, Mannell et al. coated the LV-nanoparticle constructs with magnetic microbubbles for enlargement. Upon intravenous delivery, the LV magnetic microbubbles were first trapped at the site of interest. Next ultrasound mediated destruction of the microbubbles resulted in fast release of the LVs at the site of interest with high transduction efficiency without the cost of higher cytotoxicity (Mannell, Pircher et al. 2012).

In conclusion, there seem to be some general prerequisites for successful transductional targeting of LVs: (1) use envelope gps with defined receptor binding sites, (2) abolish the natural recognition sites of the attachment gp, (3) separate fusion and attachment functions over two different molecules, (4) avoid the construction of large fusion constructs since their fusogenic capacities can be severely compromised and (5) avoid the use of immunogenic gps (Buchholz, Muhlebach et al. 2009).

\section{Genomic targeting}

Nowadays, LVs have become valuable tools for the treatment of several monogenic disorders such as hemophilia B, $\beta$-thalassemia and X-linked adrenoleukodystrophy (Cartier, Hacein-Bey-Abina et al. 2012; Payen, Colomb et al. 2012). However, the use of viral vectors that integrate their cargo into the genome of the host cell can trigger oncogenesis by insertional mutagenesis. This is exemplified by the incident where two out of 11 patients treated with a $\gamma$-retroviral vector to correct X-linked SCID, developed leukemia. This was caused by the $\gamma$ retroviral construct's tendency to insert into active genes, in this case the LMO-2 oncogene (Marshall 2002). Later on, using the same vector type to treat chronic granulomatous disease, genomic instability and myelodysplasia was observed (Stein, Ott et al. 2010). These incidents prompted substantial research into design, safety testing and optimization of integrating vectors. Thus far several measures have been taken to pose a reduced risk on insertional mutagenesis such as the development of SIN LVs containing a moderate cellular promoter (Modlich and Baum 2009; Montini, Cesana et al. 2009). Furthermore LVs are intrinsically less genotoxic than their retroviral counterparts (Montini, Cesana et al. 2006). Nevertheless, LVs have a higher transduction efficiency, which could counterbalance the reduced risk of mutagenic vector integration into the patient's genome. In addition, accumulating studies report the concept of LV-induced clonal dominance related to growth and/or survival advantage e.g. induced by vector integration and subsequent formation of aberrantly spliced mRNA forms (Fehse and Roeder 2008; Cavazzana-Calvo, Payen et al. 2010; Cesana, Sgualdino et al. 2012; Moiani, Paleari et al. 2012). In an extensive analysis to explore the effect of promoter-enhancer selection on efficacy and safety of LVs, no clear underlying mechanism could be provided for the observed. They concluded that other ill-defined risk factors must be involved for oncogenesis, including replicative stress (Ginn, Liao et al. 2010). 
Finally, next to transcriptional activation of neighboring genes, also transcriptional shut off of the tg has been reported. This was due to chromatin remodeling at the site of insertion and cessation of the therapeutic effect (Stein, Ott et al. 2010).

Therefore additional strategies have been considered to reduce the side effects related to random insertion. The most straightforward strategy is to prevent integration of the proviral cargo by the use of IDLVs. These IDLVs are produced with a mutated integrase, which results in prevention of proviral integration and generation of increased levels of circular vector episomes within the infected cells. They appear to be safer with only a 0,1 to $2,3 \%$ chance that the episomal transcript gets integrated without a marked loss in effectiveness in terms of immune stimulatory potential of the IDLV-based vaccines (Vargas, Gusella et al. 2004; Philippe, Sarkis et al. 2006; Karwacz, Mukherjee et al. 2009; Wanisch and Yanez-Munoz 2009). However, as the lentiviral episomes lack replication signals, they are gradually lost by dilution in dividing cells and only stable in quiescent cells, which is undesirable for permanent correction of any genetic disorder. Furthermore also lower tg expression levels have been reported compared to integrative vectors (Bayer, Kantor et al. 2008). Therefore several alternative strategies have been brought forward to target the integrative process to a specific 'safe' genomic site.

In a first attempt, several groups tried to fuse a heterologous DNA binding domain directly to the integrase. Bushman et al. were the first to evaluate the activity of a hybrid, composed of the HIV-1 integrase and the lambda repressor. They reported on integration primarily near the lambda operator sites on the same face of the $\beta$-DNA helix (Bushman 1994). Later a model system was used were the integrase, derived from the avian sarcoma virus or HIV-1 respectively was fused to the Escherichia coli LexA repressor protein DNA binding domain (Katz, Merkel et al. 1996; Holmes-Son and Chow 2000). When this construct was packaged into the virion in trans either by replacing the original integrase gene or by cloning it adjacent to the HIV-1 accessory protein Vpr, they observed that this enhanced the use of integration sites adjacent to the lexA operators. In another study, the HIV-1 derived integrase was fused to a synthetic polydactyl zinc finger protein E2C, which binds specifically to a contiguous 18 bp E2C recognition site (Tan, Dong et al. 2006). Although in all studies clearly a higher preference for integration near the target sequence of choice was observed, this also implicated reduced DNA-binding specificity of the fusion protein with associated decrease of integration frequency of about 80 percent compared to viruses containing wild type integrase. Furthermore this strategy is also limited by the difficulty to incorporate the fusion protein into infectious virions (Michel, Yu et al. 2010).

Another strategy is targeting the integration away from genes using tethering domains linked to the host cell-encoded transcriptional co-activator lens epithelium-derived growth factor/p75 (LEDGF/p75), a cellular integrase binding protein. For example the LEDGF/p75 chromatin interaction-binding domain has been replaced with CBX1, which binds histone $\mathrm{H} 3$ di- or trimethylated on $\mathrm{K} 9$. Subsequently proviral integration was directed to pericentric heterochromatin and intergenic regions (Llano, Vanegas et al. 2006; Ferris, Wu et al. 2010; Gijsbers, Ronen et al. 2010; Silvers, Smith et al. 2010). As this requires engineering of a host cell protein, it is not feasible for clinical applications at the present stage (Izmiryan, Basma- 
ciogullari et al. 2011). Site-specific proviral integration can also be mediated by the use of site-specific recombinases. The best known are derived from the lambda integrase family of enzymes and include the bacteriophage P1 Cre recombinase, bacteriophage lambda integrase, the yeast Flp recombinase and bacterial XerCD recombinase. They catalyze site specific recombination by a transient DNA-protein covalent linkage that brings two specific DNA repeats together (Van Duyne 2001). Depending on the orientation of the DNA repeats, the DNA segment will either be excised or inverted when in the same or opposite orientation respectively (Figure 5A, adapted from http://www.ruf.rice.edu/ rur/issue1_files/ norman.html). The Cre-loxP system has been developed for gene studies to conditionally knock out a target gene in a cell- or tissue specific manner to overcome embryonic lethality due to permanent inactivation of the target gene in an early developmental stage (Ray, Fagan et al. 2000). This system is based on two palindromic loxP sites of 34 bp that flank the gene of interest. Although these loxP sites are prevalent in the genomes of bacteriophages, they are absent in the mouse genome where they have to be introduced by targeted mutagenesis (Kos 2004). Throughout the human genome, however, loxP-like sequences or pseudo-loxP sites are present that can be recognized by either wild-type Cre or Cre variants. This last feature enables site-specific insertion of a gene in a defined loxP site in the human genome if a Cre recombinase is provided in cis or trans. Michel et al. evaluated the feasibility of combining the Cre-loxP system for gene targeting with the versatile gene delivery system of LVs for site-specific gene insertion in human cell lines. They transduced a loxP site containing cell line with a LV containing Cre recombinase in trans as a fusion protein to the HIV accessory protein Vpr. Moreover the LV contained a cassette containing a loxP site followed by the neomycin resistant gene, inserted in the U3 region of the 3'LTR. Upon reverse transcription, the loxP-neo sequence would appear in both LTRs, thereby providing a substrate for recombination that could be catalyzed by the virion-associated Vpr-Cre. Upon this recombination step, a circular product was produced that was on his turn inserted into the loxP site of the cell line, again catalyzed by virion-associated Vpr-Cre. Another example is provided by the group of Jiang et al. who demonstrated a selective inhibitory effect on the lens epithelial cells and not the retinal pigment epithelial cells (Jiang, Lu et al. 2011). Therefore they used an enhanced Cre/loxP system with a LV expressing Cre under the control of the lens-specific promoter LEP503 in combination with another LV that contained a stiffer sequence encoding eGFP with a functional polyadenylation signal between two loxP sites, followed by the HSV-TK gene, both under the control of the human phosphoglycerate kinase promoter. Expression of the downstream HSV-TK was activated by co-expression of Cre under the control of the lens-specific promoter LEP503. Although this technology allows site-specific tg insertion, there are only a limited amount of pseudo-loxP sites in the human genome and even none in the mouse genome, which makes this technique unusable for fundamental research in laboratory animals. Furthermore, two recombination events are required which has a major impact on its efficiency.

A recent strategy makes use of site-specific endonucleases to target the tg to neutral 'safe harbor' genome regions or stimulate the process of homologous recombination for gene repair (Fischer, Hacein-Bey-Abina et al. 2011). Endonucleases induce site-specific ds breaks that can be repaired by homology-directed repair, a form of homologous recombination that 
uses a copy of the genetic information from the broken DNA molecule. When the latter is provided by the same or another LV, this copy will be used to repair the ds break (Urnov, Miller et al. 2005; O'Driscoll and Jeggo 2006). The advantage of gene repair/correction is that both function and expression of the affected gene are restored while the risk associated with random vector integration is avoided. Besides the advantage of the reduced risk for insertional mutagenesis, this strategy is also used to target genes in order to knock them down or replace them with another gene by homologous recombination. The disadvantage is that the nuclease coding sequences are expressed for several days, which is not optimal for translation to the clinic due to the background off-target generation of dsDNA breaks.

One possibility is the use of the zinc finger nuclease strategy. For this, the Cys2His2 class of zinc finger DNA binding domains is engineered to recognize a DNA sequence of interest, fused to the nuclease domain of the FokI type II restriction endonuclease to yield a highly specific zinc finger (Figure 5 B, adapted from http://biol1020-2011-2.blogspot.be/2011/09/ zinc-finger-nucleases-zfn-emerging.html) (Kim, Cha et al. 1996; Pabo, Peisach et al. 2001). When two different zinc fingers are designed to bind the same sequence of interest in the opposite orientation, this will allow dimerization of the FokI domains which leads to a zinc finger induced dsDNA break (Bitinaite, Wah et al. 1998). Various strategies have been developed to engineer the Cys2His2 zinc fingers in order to bind a specific sequence either by modular assembly or by selection strategies using phage display or a cellular selection system. Naldini et al. evaluated the use of zinc finger nucleases in combination with an IDLV for gene editing. Therefore they co-transduced several cell lines with three different IDLVs, one encoding the donor sequence and two encoding the two zinc fingers (Lombardo, Genovese et al. 2007). A few years later they also used this strategy to assess zinc finger specificity genome-wide by comprehensively mapping the locations of the IDLV integration sites in cells co-transduced with GFP and zinc finger encoding LVs (Gabriel, Lombardo et al. 2011). They observed a very high efficiency and specificity, yet a measurable rate of vector integration at unidentified sites occurred with this approach, which is the sum of zinc finger mediated and background levels of IDLV integration. Moreover co-transduction with three different LVs may be a rate-limiting step in this system. Therefore the use of a single construct to express the zinc fingers and deliver the donor tg must be evaluated, especially for less permissive cells such as hematopoietic progenitors.

Another way to target the proviral genome is by the provision of a vector-associated meganuclease encoded by a separate vector or supplied as a protein within the viral particle. (Izmiryan, Basmaciogullari et al. 2011). For the latter, Ismiryan et al. fused the prototypic meganuclease I-SceI from yeast to Vpr. This avoided the potentially toxic sustained expression of the introduced endonuclease. IDLVs encoding the donor sequence and containing the meganuclease-SceI fusion construct were tested in reporter cells in which targeting events were scored by the repair of a puromycin resistance gene. They reported a two-fold higher frequency of the expected recombination event when the nuclease was delivered as a protein rather than encoded by a separate vector and therefore improved both the safety and efficacy of this LV-based gene targeting system. In conclusion, although the field of ge- 
nomic targeting is relatively new for LV-based gene therapy, it opens a tremendous amount of new possibilities.

\section{A) Cre loxP recombination}

\section{Excision Inversion}

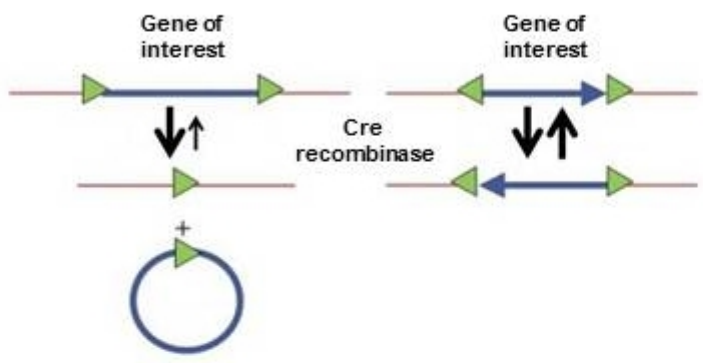

B) The zinc finger nuclease complex

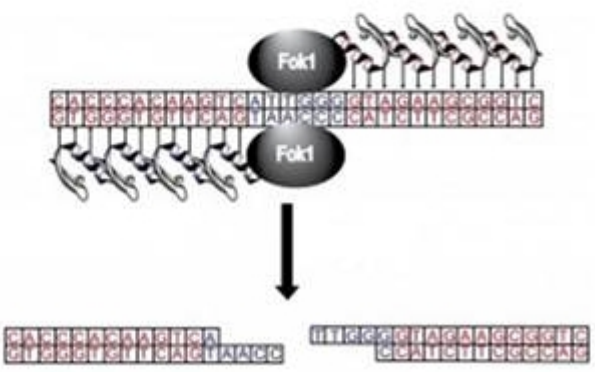

Two individual zinc finger molecules
recognize two opposite DNA strands

Dimerization of the associated Fok1 endonucleases induces a ds DNA break in between the opposite DNA sequences

Figure 5. Schematic representation of targeted genome modification by the Cre-loxP system (A) or by the zinc-finger nuclease complex (B). Cre recombinases recognize specific loxP sites in the genome, bind them en bring them together. Depending on their orientation this leads to excision (same orientation) or inversion (opposite orientation) of the sequence flanked by the two loxP sites(A). Two individual zinc finger molecules each recognize a 9 to 18 bp DNA sequence using between three and six individual zinc finger repeats that bind the major groove of DNA. The DNA sequences are non-palindromic DNA sites located respectively up -and downstream of the intended cleavage site, which is mostly about 5-7 bp long. If the zinc finger domains are perfectly specific for their intended target site then even a pair of three-finger ZFNs that recognize a total of $18 \mathrm{bp}$ can theoretically target a single locus in a mammalian genome. Next, the associated Fok1 nucleases dimerize and induce a double stranded break which can be restored by either non-homologous end-joining or homology-directed repair, which faithfully restores the original sequence by copying it from the sister chromatid or using the homologous sequence provided by a LV(B). 


\section{Concluding remarks}

LVs have proven to be efficient vehicles to deliver one or more tgs to any cell type of choice, which has led to a promising list of therapeutic applications. As the demand for experimentation in gene delivery to specific cell types increases, technologies that precisely target LVbased gene expression will become more important for research and clinical applications. Four main groups of strategies with their own possibilities as well as difficulties have been developed so far. Self-evidently, further optimization and fine-tuning of these strategies is a necessity to fulfill the expectations for targeted LV delivery in vivo. In addition to extra optimization steps, combinations of two or more of these strategies can also lead to an overall more selective, efficient and most importantly, safe LV system. Several attempts to combine the different strategies have been reported (Brown, Cantore et al. 2007; Pariente, Morizono et al. 2007; Escors and Breckpot 2011). Pariente et al. for example, reported on a LV that was transductionally targeted to prostate cancer bone metastases by a modified sindbis virus envelope that interacts with PSCA and transcriptionally targeted with a prostate cell specific promoter. This dual-targeted LV enhanced specificity to prostate cancer bone metastases after systemic delivery with respect to individual transcriptional or transductional targeting. As the developed targeting strategies already resulted in a major step forward for LV-based gene therapy, their potential will most likely be more exploited in the future, paving the way towards an all-embracing LV-based tg vehicle for the gene therapeutic field.

\section{Author details}

Cleo Goyvaerts ${ }^{1}$, Therese Liechtenstein ${ }^{2}$, Christopher Bricogne ${ }^{2}$, David Escors ${ }^{2}$ and Karine Breckpot ${ }^{1}$

1 Laboratory of Molecular and Cellular Therapy, Department of Immunology-Physiology, Vrije Universiteit Brussel, Jette, Belgium

2 Division of Infection and Immunity, Rayne Institute, University College London, London, UK

\section{References}

[1] Abruzzese, R. V., D. Godin, et al. (2000). "Ligand-dependent regulation of vascular endothelial growth factor and erythropoietin expression by a plasmid-based autoinducible GeneSwitch system." Mol Ther 2(3): 276-87.

[2] Adriani, W., F. Boyer, et al. (2010). "Social withdrawal and gambling-like profile after lentiviral manipulation of DAT expression in the rat accumbens." Int J Neuropsychopharmacol 13(10): 1329-42. 
[3] Annoni, A., M. Battaglia, et al. (2007). "The immune response to lentiviral-delivered transgene is modulated in vivo by transgene-expressing antigen-presenting cells but not by CD4+CD25+ regulatory T cells." Blood 110(6): 1788-96.

[4] Annoni, A., B. D. Brown, et al. (2009). "In vivo delivery of a microRNA-regulated transgene induces antigen-specific regulatory $\mathrm{T}$ cells and promotes immunologic tolerance." Blood 114(25): 5152-61.

[5] Azzouz, M., G. S. Ralph, et al. (2004). "VEGF delivery with retrogradely transported lentivector prolongs survival in a mouse ALS model." Nature 429(6990): 413-7.

[6] Bahi, A., F. Boyer, et al. (2004). "CD81-induced behavioural changes during chronic cocaine administration: in vivo gene delivery with regulatable lentivirus." Eur J Neurosci 19(6): 1621-33.

[7] Barde, I., M. A. Zanta-Boussif, et al. (2006). "Efficient control of gene expression in the hematopoietic system using a single Tet-on inducible lentiviral vector." Mol Ther 13(2): 382-90.

[8] Barrilleaux, B. and P. Knoepfler (2011). "Transduction of human cells with polymercomplexed ecotropic lentivirus for enhanced biosafety." J Vis Exp 2011(53).

[9] Bartel, D. P. (2004). "MicroRNAs: genomics, biogenesis, mechanism, and function." Cell 116(2): 281-97.

[10] Bauer, G., M. A. Dao, et al. (2008). "In vivo biosafety model to assess the risk of adverse events from retroviral and lentiviral vectors." Mol Ther 16(7): 1308-15.

[11] Baup, D., L. Fraga, et al. (2010). "Variegation and silencing in a lentiviral-based murine transgenic model." Transgenic Res 19(3): 399-414.

[12] Bayer, M., B. Kantor, et al. (2008). "A large U3 deletion causes increased in vivo expression from a nonintegrating lentiviral vector." Mol Ther 16(12): 1968-76.

[13] Benabdellah, K., M. Cobo, et al. (2011). "Development of an all-in-one lentiviral vector system based on the original TetR for the easy generation of Tet-ON cell lines." PLoS One 6(8): e23734.

[14] Benzekhroufa, K., B. H. Liu, et al. (2009). "Targeting central serotonergic neurons with lentiviral vectors based on a transcriptional amplification strategy." Gene Ther 16(5): 681-8.

[15] Beyer, W. R., M. Westphal, et al. (2002). "Oncoretrovirus and lentivirus vectors pseudotyped with lymphocytic choriomeningitis virus glycoprotein: generation, concentration, and broad host range." J Virol 76(3): 1488-95.

[16] Bitinaite, J., D. A. Wah, et al. (1998). "FokI dimerization is required for DNA cleavage." Proc Natl Acad Sci U S A 95(18): 10570-5.

[17] Blechacz, B. and S. J. Russell (2008). "Measles virus as an oncolytic vector platform." Curr Gene Ther 8(3): 162-75. 
[18] Blesch, A., J. Conner, et al. (2005). "Regulated lentiviral NGF gene transfer controls rescue of medial septal cholinergic neurons." Mol Ther 11(6): 916-25.

[19] Blomer, U., L. Naldini, et al. (1997). "Highly efficient and sustained gene transfer in adult neurons with a lentivirus vector." J Virol 71(9): 6641-9.

[20] Boerger, A. L., S. Snitkovsky, et al. (1999). "Retroviral vectors preloaded with a viral receptor-ligand bridge protein are targeted to specific cell types." Proc Natl Acad Sci U S A 96(17): 9867-72.

[21] Bonci, D., V. Coppola, et al. (2008). "The miR-15a-miR-16-1 cluster controls prostate cancer by targeting multiple oncogenic activities." Nat Med 14(11): 1271-7.

[22] Breckpot, K., P. Emeagi, et al. (2007). "Activation of immature monocyte-derived dendritic cells after transduction with high doses of lentiviral vectors." Hum Gene Ther 18(6): 536-46.

[23] Breckpot, K., P. U. Emeagi, et al. (2008). "Lentiviral vectors for anti-tumor immunotherapy." Curr Gene Ther 8(6): 438-48.

[24] Breckpot, K., D. Escors, et al. (2010). "HIV-1 lentiviral vector immunogenicity is mediated by Toll-like receptor 3 (TLR3) and TLR7." J Virol 84(11): 5627-36.

[25] Brown, B. D., A. Cantore, et al. (2007). "A microRNA-regulated lentiviral vector mediates stable correction of hemophilia B mice." Blood 110(13): 4144-52.

[26] Brown, B. D., B. Gentner, et al. (2007). "Endogenous microRNA can be broadly exploited to regulate transgene expression according to tissue, lineage and differentiation state." Nat Biotechnol 25(12): 1457-67.

[27] Brown, B. D. and D. Lillicrap (2002). "Dangerous liaisons: the role of "danger" signals in the immune response to gene therapy." Blood 100(4): 1133-40.

[28] Brown, B. D. and L. Naldini (2009). "Exploiting and antagonizing microRNA regulation for therapeutic and experimental applications." Nat Rev Genet 10(8): 578-85.

[29] Brown, B. D., M. A. Venneri, et al. (2006). "Endogenous microRNA regulation suppresses transgene expression in hematopoietic lineages and enables stable gene transfer." Nat Med 12(5): 585-91.

[30] Buchholz, C. J., L. J. Duerner, et al. (2008). "Retroviral display and high throughput screening." Comb Chem High Throughput Screen 11(2): 99-110.

[31] Buchholz, C. J., M. D. Muhlebach, et al. (2009). "Lentiviral vectors with measles virus glycoproteins - dream team for gene transfer?" Trends Biotechnol 27(5): 259-65.

[32] Burns, J. C., T. Friedmann, et al. (1993). "Vesicular stomatitis virus G glycoprotein pseudotyped retroviral vectors: concentration to very high titer and efficient gene transfer into mammalian and nonmammalian cells." Proc Natl Acad Sci U S A 90(17): 8033-7. 
[33] Bushman, F. D. (1994). "Tethering human immunodeficiency virus 1 integrase to a DNA site directs integration to nearby sequences." Proc Natl Acad Sci U S A 91(20): 9233-7.

[34] Calame, M., M. Cachafeiro, et al. (2011). "Retinal degeneration progression changes lentiviral vector cell targeting in the retina." PLoS One 6(8): e23782.

[35] Canivet, M., A. D. Hoffman, et al. (1990). "Replication of HIV-1 in a wide variety of animal cells following phenotypic mixing with murine retroviruses." Virology 178(2): 543-51.

[36] Cao, J., K. Sodhi, et al. (2011). "Lentiviral-human heme oxygenase targeting endothelium improved vascular function in angiotensin II animal model of hypertension." Hum Gene Ther 22(3): 271-82.

[37] Cao, O., C. Furlan-Freguia, et al. (2007). "Emerging role of regulatory T cells in gene transfer." Curr Gene Ther 7(5): 381-90.

[38] Cartier, N., S. Hacein-Bey-Abina, et al. (2012). "Lentiviral hematopoietic cell gene therapy for X-linked adrenoleukodystrophy." Methods Enzymol 507: 187-98.

[39] Cartier, N., S. Hacein-Bey-Abina, et al. (2009). "Hematopoietic stem cell gene therapy with a lentiviral vector in X-linked adrenoleukodystrophy." Science 326(5954): 818-23.

[40] Cattoglio, C., G. Facchini, et al. (2007). "Hot spots of retroviral integration in human CD34+ hematopoietic cells." Blood 110(6): 1770-8.

[41] Cavazzana-Calvo, M., S. Hacein-Bey, et al. (2000). "Gene therapy of human severe combined immunodeficiency (SCID)-X1 disease." Science 288(5466): 669-72.

[42] Cavazzana-Calvo, M., E. Payen, et al. (2010). "Transfusion independence and HMGA2 activation after gene therapy of human beta-thalassaemia." Nature 467(7313): 318-22.

[43] Cesana, D., J. Sgualdino, et al. (2012). "Whole transcriptome characterization of aberrant splicing events induced by lentiviral vector integrations." J Clin Invest 122(5): 1667-76.

[44] Chadwick, M. P., F. J. Morling, et al. (1999). "Modification of retroviral tropism by display of IGF-I." J Mol Biol 285(2): 485-94.

[45] Chandrashekran, A., M. Y. Gordon, et al. (2004). "Targeted retroviral transduction of c-kit+ hematopoietic cells using novel ligand display technology." Blood 104(9): 2697-703.

[46] Chowdhury, S., K. A. Chester, et al. (2004). "Efficient retroviral vector targeting of carcinoembryonic antigen-positive tumors." Mol Ther 9(1): 85-92.

[47] Colin, A., M. Faideau, et al. (2009). "Engineered lentiviral vector targeting astrocytes in vivo." Glia 57(6): 667-79. 
[48] Cronin, J., X. Y. Zhang, et al. (2005). "Altering the tropism of lentiviral vectors through pseudotyping." Curr Gene Ther 5(4): 387-98.

[49] Croyle, M. A., S. M. Callahan, et al. (2004). "PEGylation of a vesicular stomatitis virus G pseudotyped lentivirus vector prevents inactivation in serum." J Virol 78(2): 912-21.

[50] Cui, Y., J. Golob, et al. (2002). "Targeting transgene expression to antigen-presenting cells derived from lentivirus-transduced engrafting human hematopoietic stem/ progenitor cells." Blood 99(2): 399-408.

[51] De Palma, M., E. Montini, et al. (2005). "Promoter trapping reveals significant differences in integration site selection between MLV and HIV vectors in primary hematopoietic cells." Blood 105(6): 2307-15.

[52] De Palma, M., M. A. Venneri, et al. (2003). "In vivo targeting of tumor endothelial cells by systemic delivery of lentiviral vectors." Hum Gene Ther 14(12): 1193-206.

[53] Delenda, C. (2004). "Lentiviral vectors: optimization of packaging, transduction and gene expression." J Gene Med 6 Suppl 1: S125-38.

[54] DePolo, N. J., J. D. Reed, et al. (2000). "VSV-G pseudotyped lentiviral vector particles produced in human cells are inactivated by human serum." Mol Ther 2(3): 218-22.

[55] Deuschle, U., R. A. Hipskind, et al. (1990). "RNA polymerase II transcription blocked by Escherichia coli lac repressor." Science 248(4954): 480-3.

[56] Di Domenico, C., D. Di Napoli, et al. (2006). "Limited transgene immune response and long-term expression of human alpha-L-iduronidase in young adult mice with mucopolysaccharidosis type I by liver-directed gene therapy." Hum Gene Ther 17(11): 1112-21.

[57] Di Nunzio, F., G. Maruggi, et al. (2008). "Correction of laminin-5 deficiency in human epidermal stem cells by transcriptionally targeted lentiviral vectors." Mol Ther 16(12): 1977-85.

[58] Dreja, H. and M. Piechaczyk (2006). "The effects of N-terminal insertion into VSV-G of an scFv peptide." Virol J 3: 69.

[59] Dresch, C., S. L. Edelmann, et al. (2008). "Lentiviral-mediated transcriptional targeting of dendritic cells for induction of T cell tolerance in vivo." J Immunol 181(7): 4495-506.

[60] Dropulic, B. (2011). "Lentiviral vectors: their molecular design, safety, and use in laboratory and preclinical research." Hum Gene Ther 22(6): 649-57.

[61] Dull, T., R. Zufferey, et al. (1998). "A third-generation lentivirus vector with a conditional packaging system." J Virol 72(11): 8463-71. 
[62] Dylla, D. E., L. Xie, et al. (2011). "Altering alpha-dystroglycan receptor affinity of LCMV pseudotyped lentivirus yields unique cell and tissue tropism." Genet Vaccines Ther 9: 8.

[63] Earp, L. J., S. E. Delos, et al. (2005). "The many mechanisms of viral membrane fusion proteins." Curr Top Microbiol Immunol 285: 25-66.

[64] Ebert, M. S., J. R. Neilson, et al. (2007). "MicroRNA sponges: competitive inhibitors of small RNAs in mammalian cells." Nat Methods 4(9): 721-6.

[65] Efrat, S., D. Fusco-DeMane, et al. (1995). "Conditional transformation of a pancreatic beta-cell line derived from transgenic mice expressing a tetracycline-regulated oncogene." Proc Natl Acad Sci U S A 92(8): 3576-80.

[66] Emi, N., T. Friedmann, et al. (1991). "Pseudotype formation of murine leukemia virus with the G protein of vesicular stomatitis virus." J Virol 65(3): 1202-7.

[67] Enkirch, T., S. Kneissl, et al. (2012). "Targeted lentiviral vectors pseudotyped with the Tupaia paramyxovirus glycoproteins." Gene Ther.

[68] Escors, D. and K. Breckpot (2010). "Lentiviral vectors in gene therapy: their current status and future potential." Arch Immunol Ther Exp (Warsz) 58(2): 107-19.

[69] Escors, D. and K. Breckpot (2011). "Lentiviral vectors in gene therapy: their current status and future potential." Arch Immunol Ther Exp (Warsz) 58(2): 107-19.

[70] Fehse, B. and I. Roeder (2008). "Insertional mutagenesis and clonal dominance: biological and statistical considerations." Gene Ther 15(2): 143-53.

[71] Ferris, A. L., X. Wu, et al. (2010). "Lens epithelium-derived growth factor fusion proteins redirect HIV-1 DNA integration." Proc Natl Acad Sci U S A 107(7): 3135-40.

[72] Fielding, A. K., M. Maurice, et al. (1998). "Inverse targeting of retroviral vectors: selective gene transfer in a mixed population of hematopoietic and nonhematopoietic cells." Blood 91(5): 1802-9.

[73] Fischer, A., S. Hacein-Bey-Abina, et al. (2011). "Gene therapy for primary adaptive immune deficiencies." J Allergy Clin Immunol 127(6): 1356-9.

[74] Frecha, C., C. Costa, et al. (2012). "A novel lentiviral vector targets gene transfer into human hematopoietic stem cells in marrow from patients with bone marrow failure syndrome and in vivo in humanized mice." Blood 119(5): 1139-50.

[75] Frecha, C., F. Fusil, et al. (2011). "In vivo gene delivery into hCD34+ cells in a humanized mouse model." Methods Mol Biol 737: 367-90.

[76] Frecha, C., C. Levy, et al. (2010). "Advances in the field of lentivector-based transduction of T and B lymphocytes for gene therapy." Mol Ther 18(10): 1748-57.

[77] Frecha, C., C. Levy, et al. (2011). "Measles virus glycoprotein-pseudotyped lentiviral vector-mediated gene transfer into quiescent lymphocytes requires binding to both SLAM and CD46 entry receptors." J Virol 85(12): 5975-85. 
[78] Friedrich, R. I., K. Nopora, et al. (2012). "Transcriptional targeting of B cells with viral vectors." European Journal of Cell Biology 91(1): 86-96.

[79] Froelich, S., L. Ziegler, et al. (2009). "Targeted gene delivery to CD117-expressing cells in vivo with lentiviral vectors co-displaying stem cell factor and a fusogenic molecule." Biotechnol Bioeng 104(1): 206-15.

[80] Funke, S., A. Maisner, et al. (2008). "Targeted cell entry of lentiviral vectors." Mol Ther 16(8): 1427-36.

[81] Funke, S., I. C. Schneider, et al. (2009). "Pseudotyping lentiviral vectors with the wild-type measles virus glycoproteins improves titer and selectivity." Gene Ther 16(5): 700-5.

[82] Furth, P. A., L. St Onge, et al. (1994). "Temporal control of gene expression in transgenic mice by a tetracycline-responsive promoter." Proc Natl Acad Sci U S A 91(20): 9302-6.

[83] Fussenegger, M., R. P. Morris, et al. (2000). "Streptogramin-based gene regulation systems for mammalian cells." Nat Biotechnol 18(11): 1203-8.

[84] Gabriel, R., A. Lombardo, et al. (2011). "An unbiased genome-wide analysis of zincfinger nuclease specificity." Nat Biotechnol 29(9): 816-23.

[85] Gainkam, L. O., L. Huang, et al. (2008). "Comparison of the biodistribution and tumor targeting of two 99mTc-labeled anti-EGFR nanobodies in mice, using pinhole SPECT/micro-CT." J Nucl Med 49(5): 788-95.

[86] Galimi, F., E. Saez, et al. (2005). "Development of ecdysone-regulated lentiviral vectors." Mol Ther 11(1): 142-8.

[87] Gascon, S., J. A. Paez-Gomez, et al. (2008). "Dual-promoter lentiviral vectors for constitutive and regulated gene expression in neurons." J Neurosci Methods 168(1): 104-12.

[88] Gaspar, H. B., K. L. Parsley, et al. (2004). "Gene therapy of X-linked severe combined immunodeficiency by use of a pseudotyped gammaretroviral vector." Lancet 364(9452): 2181-7.

[89] Gennari, F., L. Lopes, et al. (2009). "Single-chain antibodies that target lentiviral vectors to MHC class II on antigen-presenting cells." Hum Gene Ther 20(6): 554-62.

[90] Gentner, B., G. Schira, et al. (2009). "Stable knockdown of microRNA in vivo by lentiviral vectors." Nat Methods 6(1): 63-6.

[91] Gentner, B., I. Visigalli, et al. (2010). "Identification of hematopoietic stem cell-specific miRNAs enables gene therapy of globoid cell leukodystrophy." Sci Transl Med 2(58): 58ra84.

[92] Gijsbers, R., K. Ronen, et al. (2010). "LEDGF hybrids efficiently retarget lentiviral integration into heterochromatin." Mol Ther 18(3): 552-60. 
[93] Gilham, D. E., A. L. M. Lie, et al. (2010). "Cytokine stimulation and the choice of promoter are critical factors for the efficient transduction of mouse T cells with HIV-1 vectors." J Gene Med 12(2): 129-36.

[94] Ginn, S. L., S. H. Liao, et al. (2010). "Lymphomagenesis in SCID-X1 mice following lentivirus-mediated phenotype correction independent of insertional mutagenesis and gammac overexpression." Mol Ther 18(5): 965-76.

[95] Gossen, M. and H. Bujard (1992). "Tight control of gene expression in mammalian cells by tetracycline-responsive promoters." Proc Natl Acad Sci U S A 89(12): 5547-51.

[96] Goyvaerts, C., K. De Groeve, et al. (2012). "Development of the Nanobody display technology to target lentiviral vectors to antigen-presenting cells." Gene Ther.

[97] Guibinga, G. H., F. L. Hall, et al. (2004). "Ligand-modified vesicular stomatitis virus glycoprotein displays a temperature-sensitive intracellular trafficking and virus assembly phenotype." Mol Ther 9(1): 76-84.

[98] Hamers-Casterman, C., T. Atarhouch, et al. (1993). "Naturally occurring antibodies devoid of light chains." Nature 363(6428): 446-8.

[99] Hanawa, H., D. A. Persons, et al. (2002). "High-level erythroid lineage-directed gene expression using globin gene regulatory elements after lentiviral vector-mediated gene transfer into primitive human and murine hematopoietic cells." Hum Gene Ther 13(17): 2007-16.

[100] Hawley, T. S., A. Z. Fong, et al. (1998). "Leukemic predisposition of mice transplanted with gene-modified hematopoietic precursors expressing flt3 ligand." Blood 92(6): 2003-11.

[101] Heckl, D., D. C. Wicke, et al. (2011). "Lentiviral gene transfer regenerates hematopoietic stem cells in a mouse model for Mpl-deficient aplastic anemia." Blood 117(14): 3737-47.

[102] Herold, M. J., J. van den Brandt, et al. (2008). "Inducible and reversible gene silencing by stable integration of an shRNA-encoding lentivirus in transgenic rats." Proc Natl Acad Sci U S A 105(47): 18507-12.

[103] Hioki, H., H. Kameda, et al. (2007). "Efficient gene transduction of neurons by lentivirus with enhanced neuron-specific promoters." Gene Ther 14(11): 872-82.

[104] Hioki, H., E. Kuramoto, et al. (2009). "High-level transgene expression in neurons by lentivirus with Tet-Off system." Neurosci Res 63(2): 149-54.

[105] Hofmann, H., K. Hattermann, et al. (2004). "S protein of severe acute respiratory syndrome-associated coronavirus mediates entry into hepatoma cell lines and is targeted by neutralizing antibodies in infected patients." J Virol 78(12): 6134-42.

[106] Holmes-Son, M. L. and S. A. Chow (2000). "Integrase-lexA fusion proteins incorporated into human immunodeficiency virus type 1 that contains a catalytically inactive integrase gene are functional to mediate integration." J Virol 74(24): 11548-56. 
[107] Hsieh, Y. J., F. D. Chen, et al. (2011). "The EIIAPA Chimeric Promoter for Tumor Specific Gene Therapy of Hepatoma." Mol Imaging Biol.

[108] Hu, B., B. Dai, et al. (2010). "Vaccines delivered by integration-deficient lentiviral vectors targeting dendritic cells induces strong antigen-specific immunity." Vaccine 28(41): 6675-83.

[109] Izmiryan, A., S. Basmaciogullari, et al. (2011). "Efficient gene targeting mediated by a lentiviral vector-associated meganuclease." Nucleic Acids Res 39(17): 7610-9.

[110] Jakobsson, J., C. Ericson, et al. (2003). "Targeted transgene expression in rat brain using lentiviral vectors." J Neurosci Res 73(6): 876-85.

[111] Jiang, Y. X., Y. Lu, et al. (2011). "Using HSV-TK/GCV suicide gene therapy to inhibit lens epithelial cell proliferation for treatment of posterior capsular opacification." Mol Vis 17: 291-9.

[112] Kang, Y., C. S. Stein, et al. (2002). "In vivo gene transfer using a nonprimate lentiviral vector pseudotyped with Ross River Virus glycoproteins." J Virol 76(18): 9378-88.

[113] Karavanas, G., M. Marin, et al. (2002). "The insertion of an anti-MHC I ScFv into the N-terminus of an ecotropic MLV glycoprotein does not alter its fusiogenic potential on murine cells." Virus Res 83(1-2): 57-69.

[114] Karwacz, K., S. Mukherjee, et al. (2009). "Nonintegrating lentivector vaccines stimulate prolonged T-cell and antibody responses and are effective in tumor therapy." J Virol 83(7): 3094-103.

[115] Katane, M., E. Takao, et al. (2002). "Factors affecting the direct targeting of murine leukemia virus vectors containing peptide ligands in the envelope protein." EMBO Rep 3(9): 899-904.

[116] Katz, R. A., G. Merkel, et al. (1996). "Targeting of retroviral integrase by fusion to a heterologous DNA binding domain: in vitro activities and incorporation of a fusion protein into viral particles." Virology 217(1): 178-90.

[117] Kelly, E. J., E. M. Hadac, et al. (2008). "Engineering microRNA responsiveness to decrease virus pathogenicity." Nat Med 14(11): 1278-83.

[118] Kerns, H. M., B. Y. Ryu, et al. (2010). "B cell-specific lentiviral gene therapy leads to sustained B-cell functional recovery in a murine model of X-linked agammaglobulinemia." Blood 115(11): 2146-55.

[119] Kim, S., G. J. Kim, et al. (2007). "Efficiency of the elongation factor-1alpha promoter in mammalian embryonic stem cells using lentiviral gene delivery systems." Stem Cells Dev 16(4): 537-45.

[120] Kim, Y. G., J. Cha, et al. (1996). "Hybrid restriction enzymes: zinc finger fusions to Fok I cleavage domain." Proc Natl Acad Sci U S A 93(3): 1156-60. 
[121] Kimura, T., R. C. Koya, et al. (2007). "Lentiviral vectors with CMV or MHCII promoters administered in vivo: immune reactivity versus persistence of expression." Mol Ther 15(7): 1390-9.

[122] Kobinger, G. P., S. Deng, et al. (2004). "Transduction of human islets with pseudotyped lentiviral vectors." Hum Gene Ther 15(2): 211-9.

[123] Kobinger, G. P., D. J. Weiner, et al. (2001). "Filovirus-pseudotyped lentiviral vector can efficiently and stably transduce airway epithelia in vivo." Nat Biotechnol 19(3): 225-30.

[124] Kos, C. H. (2004). "Cre/loxP system for generating tissue-specific knockout mouse models." Nutr Rev 62(6 Pt 1): 243-6.

[125] Kueng, H. J., V. M. Leb, et al. (2007). "General strategy for decoration of enveloped viruses with functionally active lipid-modified cytokines." J Virol 81(16): 8666-76.

[126] Kumar, M. S., S. J. Erkeland, et al. (2008). "Suppression of non-small cell lung tumor development by the let-7 microRNA family." Proc Natl Acad Sci U S A 105(10): 3903-8.

[127] Kuroda, H., R. H. Kutner, et al. (2008). "A comparative analysis of constitutive and cell-specific promoters in the adult mouse hippocampus using lentivirus vectormediated gene transfer." J Gene Med 10(11): 1163-75.

[128] Lachmann, N., J. Jagielska, et al. (2011). "MicroRNA-150-regulated vectors allow lymphocyte-sparing transgene expression in hematopoietic gene therapy." Gene Ther.

[129] Lai, E. C. (2002). "Micro RNAs are complementary to 3' UTR sequence motifs that mediate negative post-transcriptional regulation." Nat Genet 30(4): 363-4.

[130] Lakso, M., B. Sauer, et al. (1992). "Targeted oncogene activation by site-specific recombination in transgenic mice." Proc Natl Acad Sci U S A 89(14): 6232-6.

[131] Latta-Mahieu, M., M. Rolland, et al. (2002). "Gene transfer of a chimeric trans-activator is immunogenic and results in short-lived transgene expression." Hum Gene Ther 13(13): 1611-20.

[132] Lavillette, D., S. J. Russell, et al. (2001). "Retargeting gene delivery using surface-engineered retroviral vector particles." Curr Opin Biotechnol 12(5): 461-6.

[133] Lee, C. J., X. Fan, et al. (2011). "Promoter-specific lentivectors for long-term, cardiacdirected therapy of Fabry disease." J Cardiol 57(1): 115-22.

[134] Lee, C. L., J. Dang, et al. (2011). "Engineered lentiviral vectors pseudotyped with a CD4 receptor and a fusogenic protein can target cells expressing HIV-1 envelope proteins." Virus Res 160(1-2): 340-50.

[135] Lei, Y., K. I. Joo, et al. (2009). "Engineering fusogenic molecules to achieve targeted transduction of enveloped lentiviral vectors." J Biol Eng 3: 8. 
[136] Leuci, V., L. Gammaitoni, et al. (2009). "Efficient transcriptional targeting of human hematopoietic stem cells and blood cell lineages by lentiviral vectors containing the regulatory element of the Wiskott-Aldrich syndrome gene." Stem Cells 27(11): 2815-23.

[137] Li, M., N. Husic, et al. (2010). "Optimal promoter usage for lentiviral vector-mediated transduction of cultured central nervous system cells." J Neurosci Methods 189(1): 56-64.

[138] Lin, A. H., N. Kasahara, et al. (2001). "Receptor-specific targeting mediated by the coexpression of a targeted murine leukemia virus envelope protein and a binding-defective influenza hemagglutinin protein." Hum Gene Ther 12(4): 323-32.

[139] Liu, B., J. F. Paton, et al. (2008). "Viral vectors based on bidirectional cell-specific mammalian promoters and transcriptional amplification strategy for use in vitro and in vivo." BMC Biotechnol 8: 49.

[140] Liu, B., S. Wang, et al. (2008). "Enhancement of cell-specific transgene expression from a Tet-Off regulatory system using a transcriptional amplification strategy in the rat brain." J Gene Med 10(5): 583-92.

[141] Liu, B. H., X. Wang, et al. (2004). "CMV enhancer/human PDGF-beta promoter for neuron-specific transgene expression." Gene Ther 11(1): 52-60.

[142] Llano, M., M. Vanegas, et al. (2006). "Identification and characterization of the chromatin-binding domains of the HIV-1 integrase interactor LEDGF/p75." J Mol Biol 360(4): 760-73.

[143] Lodish, H. F. and R. A. Weiss (1979). "Selective isolation of mutants of vesicular stomatitis virus defective in production of the viral glycoprotein." J Virol 30(1): 177-89.

[144] Lombardo, A., P. Genovese, et al. (2007). "Gene editing in human stem cells using zinc finger nucleases and integrase-defective lentiviral vector delivery." Nat Biotechnol 25(11): 1298-306.

[145] Lopes, L., M. Dewannieux, et al. (2008). "Immunization with a lentivector that targets tumor antigen expression to dendritic cells induces potent CD8+ and CD4+ T-cell responses." J Virol 82(1): 86-95.

[146] Lopes, L., M. Dewannieux, et al. (2011). "A lentiviral vector pseudotype suitable for vaccine development." J Gene Med 13(3): 181-7.

[147] Lopez-Ornelas, A., T. Mejia-Castillo, et al. (2011). "Lentiviral transfer of an inducible transgene expressing a soluble form of Gas1 causes glioma cell arrest, apoptosis and inhibits tumor growth." Cancer Gene Ther 18(2): 87-99.

[148] Manilla, P., T. Rebello, et al. (2005). "Regulatory considerations for novel gene therapy products: a review of the process leading to the first clinical lentiviral vector." Hum Gene Ther 16(1): 17-25. 
[149] Mannell, H., J. Pircher, et al. (2012). "Targeted endothelial gene delivery by ultrasonic destruction of magnetic microbubbles carrying lentiviral vectors." Pharm Res 29(5): 1282-94.

[150] Marshall, E. (2002). "Gene therapy. What to do when clear success comes with an unclear risk?" Science 298(5593): 510-1.

[151] Matrai, J., A. Cantore, et al. (2011). "Hepatocyte-targeted expression by integrase-defective lentiviral vectors induces antigen-specific tolerance in mice with low genotoxic risk." Hepatology 53(5): 1696-707.

[152] Matsui, H., C. Hegadorn, et al. (2011). "A microRNA-regulated and GP64-pseudotyped lentiviral vector mediates stable expression of FVIII in a murine model of Hemophilia A." Mol Ther 19(4): 723-30.

[153] Maurice, M., S. Mazur, et al. (1999). "Efficient gene delivery to quiescent interleukin-2 (IL-2)-dependent cells by murine leukemia virus-derived vectors harboring IL-2 chimeric envelope glycoproteins." Blood 94(2): 401-10.

[154] Maurice, M., E. Verhoeyen, et al. (2002). "Efficient gene transfer into human primary blood lymphocytes by surface-engineered lentiviral vectors that display a T cell-activating polypeptide." Blood 99(7): 2342-50.

[155] Mazarakis, N. D., M. Azzouz, et al. (2001). "Rabies virus glycoprotein pseudotyping of lentiviral vectors enables retrograde axonal transport and access to the nervous system after peripheral delivery." Hum Mol Genet 10(19): 2109-21.

[156] McIver, S. R., C. S. Lee, et al. (2005). "Lentiviral transduction of murine oligodendrocytes in vivo." J Neurosci Res 82(3): 397-403.

[157] Michel, G., Y. Yu, et al. (2010). "Site-specific gene insertion mediated by a Cre-loxPcarrying lentiviral vector." Mol Ther 18(10): 1814-21.

[158] Miletic, H., Y. H. Fischer, et al. (2004). "Selective transduction of malignant glioma by lentiviral vectors pseudotyped with lymphocytic choriomeningitis virus glycoproteins." Hum Gene Ther 15(11): 1091-100.

[159] Mitomo, K., U. Griesenbach, et al. (2010). "Toward gene therapy for cystic fibrosis using a lentivirus pseudotyped with Sendai virus envelopes." Mol Ther 18(6): 1173-82.

[160] Modlich, U. and C. Baum (2009). "Preventing and exploiting the oncogenic potential of integrating gene vectors." J Clin Invest 119(4): 755-8.

[161] Moiani, A., Y. Paleari, et al. (2012). "Lentiviral vector integration in the human genome induces alternative splicing and generates aberrant transcripts." J Clin Invest 122(5): 1653-66.

[162] Montini, E., D. Cesana, et al. (2009). "The genotoxic potential of retroviral vectors is strongly modulated by vector design and integration site selection in a mouse model of HSC gene therapy." J Clin Invest 119(4): 964-75. 
[163] Montini, E., D. Cesana, et al. (2006). "Hematopoietic stem cell gene transfer in a tumor-prone mouse model uncovers low genotoxicity of lentiviral vector integration." Nat Biotechnol 24(6): 687-96.

[164] Morizono, K., A. Ku, et al. (2010). "Redirecting lentiviral vectors pseudotyped with Sindbis virus-derived envelope proteins to DC-SIGN by modification of N-linked glycans of envelope proteins." J Virol 84(14): 6923-34.

[165] Morizono, K., Y. Xie, et al. (2005). "Lentiviral vector retargeting to P-glycoprotein on metastatic melanoma through intravenous injection." Nat Med 11(3): 346-52.

[166] Munch, R. C., M. D. Muhlebach, et al. (2011). "DARPins: an efficient targeting domain for lentiviral vectors." Mol Ther 19(4): 686-93.

[167] Naldini, L., U. Blomer, et al. (1996). "In vivo gene delivery and stable transduction of nondividing cells by a lentiviral vector." Science 272(5259): 263-7.

[168] Neda, H., C. H. Wu, et al. (1991). "Chemical modification of an ecotropic murine leukemia virus results in redirection of its target cell specificity." J Biol Chem 266(22): 14143-6.

[169] Neddermann, P., C. Gargioli, et al. (2003). "A novel, inducible, eukaryotic gene expression system based on the quorum-sensing transcription factor TraR." EMBO Rep 4(2): 159-65.

[170] Nefkens, I., J. M. Garcia, et al. (2007). "Hemagglutinin pseudotyped lentiviral particles: characterization of a new method for avian H5N1 influenza sero-diagnosis." J Clin Virol 39(1): 27-33.

[171] Nelson, P., M. Kiriakidou, et al. (2003). "The microRNA world: small is mighty." Trends Biochem Sci 28(10): 534-40.

[172] Nguyen, T. H., J. C. Pages, et al. (1998). "Amphotropic retroviral vectors displaying hepatocyte growth factor-envelope fusion proteins improve transduction efficiency of primary hepatocytes." Hum Gene Ther 9(17): 2469-79.

[173] O'Driscoll, M. and P. A. Jeggo (2006). "The role of double-strand break repair - insights from human genetics." Nat Rev Genet 7(1): 45-54.

[174] O'Leary, V. B., S. V. Ovsepian, et al. (2011). "Innocuous full-length botulinum neurotoxin targets and promotes the expression of lentiviral vectors in central and autonomic neurons." Gene Ther 18(7): 656-65.

[175] Ogueta, S. B., F. Yao, et al. (2001). "Design and in vitro characterization of a single regulatory module for efficient control of gene expression in both plasmid DNA and a self-inactivating lentiviral vector." Mol Med 7(8): 569-79.

[176] Pabo, C. O., E. Peisach, et al. (2001). "Design and selection of novel Cys2His2 zinc finger proteins." Annu Rev Biochem 70: 313-40. 
[177] Page, K. A., N. R. Landau, et al. (1990). "Construction and use of a human immunodeficiency virus vector for analysis of virus infectivity." J Virol 64(11): 5270-6.

[178] Palmowski, M. J., L. Lopes, et al. (2004). "Intravenous injection of a lentiviral vector encoding NY-ESO-1 induces an effective CTL response." J Immunol 172(3): 1582-7.

[179] Papapetrou, E. P., D. Kovalovsky, et al. (2009). "Harnessing endogenous miR-181a to segregate transgenic antigen receptor expression in developing versus post-thymic $\mathrm{T}$ cells in murine hematopoietic chimeras." J Clin Invest 119(1): 157-68.

[180] Pariente, N., S. H. Mao, et al. (2008). "Efficient targeted transduction of primary human endothelial cells with dual-targeted lentiviral vectors." J Gene Med 10(3): 242-8.

[181] Pariente, N., K. Morizono, et al. (2007). "A novel dual-targeted lentiviral vector leads to specific transduction of prostate cancer bone metastases in vivo after systemic administration." Mol Ther 15(11): 1973-81.

[182] Parker, D. G., H. M. Brereton, et al. (2009). "A steroid-inducible promoter for the cornea." Br J Ophthalmol 93(9): 1255-9.

[183] Payen, E., C. Colomb, et al. (2012). "Lentivirus vectors in beta-thalassemia." Methods Enzymol 507: 109-24.

[184] Petrigliano, F. A., M. S. Virk, et al. (2009). "Targeting of prostate cancer cells by a cytotoxic lentiviral vector containing a prostate stem cell antigen (PSCA) promoter." Prostate 69(13): 1422-34.

[185] Peviani, M., M. Kurosaki, et al. (2012). "Lentiviral vectors carrying enhancer elements of $\mathrm{Hb} 9$ promoter drive selective transgene expression in mouse spinal cord motor neurons." J Neurosci Methods 205(1): 139-47.

[186] Philippe, S., C. Sarkis, et al. (2006). "Lentiviral vectors with a defective integrase allow efficient and sustained transgene expression in vitro and in vivo." Proc Natl Acad Sci U S A 103(47): 17684-9.

[187] Pollock, R., R. Issner, et al. (2000). "Delivery of a stringent dimerizer-regulated gene expression system in a single retroviral vector." Proc Natl Acad Sci U S A 97(24): 13221-6.

[188] Ramezani, A. and R. G. Hawley (2002). "Overview of the HIV-1 Lentiviral Vector System." Curr Protoc Mol Biol Chapter 16: Unit 1621.

[189] Rasko, J. E., J. L. Battini, et al. (1999). "The RD114/simian type D retrovirus receptor is a neutral amino acid transporter." Proc Natl Acad Sci U S A 96(5): 2129-34.

[190] Ray, M. K., S. P. Fagan, et al. (2000). "The Cre-loxP system: a versatile tool for targeting genes in a cell- and stage-specific manner." Cell Transplant 9(6): 805-15.

[191] Reiser, J., Z. Lai, et al. (2000). "Development of multigene and regulated lentivirus vectors." J Virol 74(22): 10589-99. 
[192] Roberts, M. C. (2002). "Resistance to tetracycline, macrolide-lincosamide-streptogramin, trimethoprim, and sulfonamide drug classes." Mol Biotechnol 20(3): 261-83.

[193] Roet, K. C., R. Eggers, et al. (2012). "Non-invasive bioluminescence imaging of olfactory ensheathing glia and Schwann cells following transplantation into the lesioned rat spinal cord." Cell Transplant.

[194] Romano, G., P. P. Claudio, et al. (2003). "Human immunodeficiency virus type 1 (HIV-1) derived vectors: safety considerations and controversy over therapeutic applications." Eur J Dermatol 13(5): 424-9.

[195] Roux, P., P. Jeanteur, et al. (1989). "A versatile and potentially general approach to the targeting of specific cell types by retroviruses: application to the infection of human cells by means of major histocompatibility complex class I and class II antigens by mouse ecotropic murine leukemia virus-derived viruses." Proc Natl Acad Sci U S A 86(23): 9079-83.

[196] Sachdeva, R., M. E. Jonsson, et al. (2010). "Tracking differentiating neural progenitors in pluripotent cultures using microRNA-regulated lentiviral vectors." Proc Natl Acad Sci U S A 107(25): 11602-7.

[197] Sakuma, T., S. S. De Ravin, et al. (2010). "Characterization of retroviral and lentiviral vectors pseudotyped with xenotropic murine leukemia virus-related virus envelope glycoprotein." Hum Gene Ther 21(12): 1665-73.

[198] Sanchez-Danes, A., A. Consiglio, et al. (2012). "Efficient generation of A9 midbrain dopaminergic neurons by lentiviral delivery of LMX1A in human embryonic stem cells and induced pluripotent stem cells." Hum Gene Ther 23(1): 56-69.

[199] Sarkar, N. N. (2002). "Mifepristone: bioavailability, pharmacokinetics and use-effectiveness." Eur J Obstet Gynecol Reprod Biol 101(2): 113-20.

[200] Sayed, D., S. Rane, et al. (2008). "MicroRNA-21 targets Sprouty2 and promotes cellular outgrowths." Mol Biol Cell 19(8): 3272-82.

[201] Schaffer, D. V., J. T. Koerber, et al. (2008). "Molecular engineering of viral gene delivery vehicles." Annu Rev Biomed Eng 10: 169-94.

[202] Scherr, M., L. Venturini, et al. (2007). "Lentivirus-mediated antagomir expression for specific inhibition of miRNA function." Nucleic Acids Res 35(22): e149.

[203] Semple-Rowland, S. L., W. E. Coggin, et al. (2010). "Expression characteristics of dual-promoter lentiviral vectors targeting retinal photoreceptors and Muller cells." Mol Vis 16: 916-34.

[204] Semple-Rowland, S. L., K. S. Eccles, et al. (2007). "Targeted expression of two proteins in neural retina using self-inactivating, insulated lentiviral vectors carrying two internal independent promoters." Mol Vis 13: 2001-11.

[205] Seo, E., S. Kim, et al. (2009). "Induction of cancer cell-specific death via MMP2 promoterdependent Bax expression." BMB Rep 42(4): 217-22. 
[206] Shinoda, Y., K. Hieda, et al. (2009). "Efficient transduction of cytotoxic and antiHIV-1 genes by a gene-regulatable lentiviral vector." Virus Genes 39(2): 165-75.

[207] Silvers, R. M., J. A. Smith, et al. (2010). "Modification of integration site preferences of an HIV-1-based vector by expression of a novel synthetic protein." Hum Gene Ther 21(3): 337-49.

[208] Singhal, R., X. Deng, et al. (2011). "Long-distance effects of insertional mutagenesis." PLoS One 6(1): e15832.

[209] Sirin, O. and F. Park (2003). "Regulating gene expression using self-inactivating lentiviral vectors containing the mifepristone-inducible system." Gene 323: 67-77.

[210] Somia, N. V., H. Miyoshi, et al. (2000). "Retroviral vector targeting to human immunodeficiency virus type 1-infected cells by receptor pseudotyping." J Virol 74(9): 4420-4.

[211] Steffens, S., J. Tebbets, et al. (2004). "Transduction of human glial and neuronal tumor cells with different lentivirus vector pseudotypes." J Neurooncol 70(3): 281-8.

[212] Steidl, S., J. Stitz, et al. (2002). "Coreceptor Switch of [MLV(SIVagm)] pseudotype vectors by V3-loop exchange." Virology 300(2): 205-16.

[213] Stein, C. S., I. Martins, et al. (2005). "The lymphocytic choriomeningitis virus envelope glycoprotein targets lentiviral gene transfer vector to neural progenitors in the murine brain." Mol Ther 11(3): 382-9.

[214] Stein, S., M. G. Ott, et al. (2010). "Genomic instability and myelodysplasia with monosomy 7 consequent to EVI1 activation after gene therapy for chronic granulomatous disease." Nat Med 16(2): 198-204.

[215] Stitz, J., C. J. Buchholz, et al. (2000). "Lentiviral vectors pseudotyped with envelope glycoproteins derived from gibbon ape leukemia virus and murine leukemia virus 10A1." Virology 273(1): 16-20.

[216] Strang, B. L., Y. Ikeda, et al. (2004). "Characterization of HIV-1 vectors with gammaretrovirus envelope glycoproteins produced from stable packaging cells." Gene Ther 11(7): 591-8.

[217] Strang, B. L., Y. Takeuchi, et al. (2005). "Human immunodeficiency virus type 1 vectors with alphavirus envelope glycoproteins produced from stable packaging cells." J Virol 79(3): 1765-71.

[218] Szulc, J., M. Wiznerowicz, et al. (2006). "A versatile tool for conditional gene expression and knockdown." Nat Methods 3(2): 109-16.

[219] Tai, A., S. Froelich, et al. (2011). "Production of lentiviral vectors with enhanced efficiency to target dendritic cells by attenuating mannosidase activity of mammalian cells." J Biol Eng 5(1): 1.

[220] Tan, W., Z. Dong, et al. (2006). "Human immunodeficiency virus type 1 incorporated with fusion proteins consisting of integrase and the designed polydactyl zinc finger 
protein E2C can bias integration of viral DNA into a predetermined chromosomal region in human cells." J Virol 80(4): 1939-48.

[221] Tian, J., P. Lei, et al. (2008). "Regulated insulin delivery from human epidermal cells reverses hyperglycemia." Mol Ther 16(6): 1146-53.

[222] Toniatti, C., H. Bujard, et al. (2004). "Gene therapy progress and prospects: transcription regulatory systems." Gene Ther 11(8): 649-57.

[223] Uch, R., R. Gerolami, et al. (2003). "Hepatoma cell-specific ganciclovir-mediated toxicity of a lentivirally transduced HSV-TkEGFP fusion protein gene placed under the control of rat alpha-fetoprotein gene regulatory sequences." Cancer Gene Ther 10(9): 689-95.

[224] Urnov, F. D., J. C. Miller, et al. (2005). "Highly efficient endogenous human gene correction using designed zinc-finger nucleases." Nature 435(7042): 646-51.

[225] Valastyan, S., F. Reinhardt, et al. (2009). "A pleiotropically acting microRNA, miR-31, inhibits breast cancer metastasis." Cell 137(6): 1032-46.

[226] Van Duyne, G. D. (2001). "A structural view of cre-loxp site-specific recombination." Annu Rev Biophys Biomol Struct 30: 87-104.

[227] Vandendriessche, T., L. Thorrez, et al. (2007). "Efficacy and safety of adeno-associated viral vectors based on serotype 8 and 9 vs. lentiviral vectors for hemophilia B gene therapy." J Thromb Haemost 5(1): 16-24.

[228] Vaneycken, I., N. Devoogdt, et al. (2011). "Preclinical screening of anti-HER2 nanobodies for molecular imaging of breast cancer." FASEB J 25(7): 2433-46.

[229] Vargas, J., Jr., G. L. Gusella, et al. (2004). "Novel integrase-defective lentiviral episomal vectors for gene transfer." Hum Gene Ther 15(4): 361-72.

[230] Verhoeyen, E., V. Dardalhon, et al. (2003). "IL-7 surface-engineered lentiviral vectors promote survival and efficient gene transfer in resting primary T lymphocytes." Blood 101(6): 2167-74.

[231] Verhoeyen, E., M. Wiznerowicz, et al. (2005). "Novel lentiviral vectors displaying "early-acting cytokines" selectively promote survival and transduction of NOD/SCID repopulating human hematopoietic stem cells." Blood 106(10): 3386-95.

[232] Waehler, R., S. J. Russell, et al. (2007). "Engineering targeted viral vectors for gene therapy." Nat Rev Genet 8(8): 573-87.

[233] Wang, Y., H. H. Hu, et al. (2012). "Lentiviral transgenic microRNA-based shRNA suppressed mouse cytochromosome P450 3A (CYP3A) expression in a dose-dependent and inheritable manner." PLoS One 7(1): e30560.

[234] Wanisch, K. and R. J. Yanez-Munoz (2009). "Integration-deficient lentiviral vectors: a slow coming of age." Mol Ther 17(8): 1316-32. 
[235] Watson, D. J., G. P. Kobinger, et al. (2002). "Targeted transduction patterns in the mouse brain by lentivirus vectors pseudotyped with VSV, Ebola, Mokola, LCMV, or MuLV envelope proteins." Mol Ther 5(5 Pt 1): 528-37.

[236] Wiederschain, D., S. Wee, et al. (2009). "Single-vector inducible lentiviral RNAi system for oncology target validation." Cell Cycle 8(3): 498-504.

[237] Wong, L. F., M. Azzouz, et al. (2004). "Transduction patterns of pseudotyped lentiviral vectors in the nervous system." Mol Ther 9(1): 101-11.

[238] Xu, Z. L., H. Mizuguchi, et al. (2003). "Regulated gene expression from adenovirus vectors: a systematic comparison of various inducible systems." Gene 309(2): 145-51.

[239] Yang, H., K. I. Joo, et al. (2009). "Cell type-specific targeting with surface-engineered lentiviral vectors co-displaying OKT3 antibody and fusogenic molecule." Pharm Res 26(6): 1432-45.

[240] Yang, L., H. Yang, et al. (2008). "Engineered lentivector targeting of dendritic cells for in vivo immunization." Nat Biotechnol 26(3): 326-34.

[241] Yu, D., D. Chen, et al. (2001). "Prostate-specific targeting using PSA promoter-based lentiviral vectors." Cancer Gene Ther 8(9): 628-35.

[242] Yu, D., C. Scott, et al. (2006). "Targeting and killing of prostate cancer cells using lentiviral constructs containing a sequence recognized by translation factor eIF4E and a prostate-specific promoter." Cancer Gene Ther 13(1): 32-43.

[243] Yu, S. T., C. Li, et al. (2011). "Noninvasive and real-time monitoring of the therapeutic response of tumors in vivo with an optimized hTERT promoter." Cancer 118(7): 1884-93.

[244] Zeilfelder, U. and V. Bosch (2001). "Properties of wild-type, C-terminally truncated, and chimeric maedi-visna virus glycoprotein and putative pseudotyping of retroviral vector particles." J Virol 75(1): 548-55.

[245] Zhang, J., L. Zou, et al. (2009). "Rapid generation of dendritic cell specific transgenic mice by lentiviral vectors." Transgenic Res 18(6): 921-31.

[246] Zhang, X. Y., R. H. Kutner, et al. (2010). "Cell-specific targeting of lentiviral vectors mediated by fusion proteins derived from Sindbis virus, vesicular stomatitis virus, or avian sarcoma/leukosis virus." Retrovirology 7:3.

[247] Zheng, J. Y., D. Chen, et al. (2003). "Regression of prostate cancer xenografts by a lentiviral vector specifically expressing diphtheria toxin A." Cancer Gene Ther 10(10): 764-70.

[248] Zhu, Z. H., S. S. Chen, et al. (1990). "Phenotypic mixing between human immunodeficiency virus and vesicular stomatitis virus or herpes simplex virus." J Acquir Immune Defic Syndr 3(3): 215-9. 
[249] Ziegler, L., L. Yang, et al. (2008). "Targeting lentiviral vectors to antigen-specific immunoglobulins." Hum Gene Ther 19(9): 861-72.

[250] Zufferey, R., D. Nagy, et al. (1997). "Multiply attenuated lentiviral vector achieves efficient gene delivery in vivo." Nat Biotechnol 15(9): 871-5. 